PREPARED FOR THE U.S. DEPARTMENT OF ENERGY, UNDER CONTRACT DE-AC02-76CH03073

PPPL-3836

PPPL-3836

UC-70

Circulation in a Short Cylindrical Couette System

by

Akira Kageyama, Hantao Ji, and Jeremy Goodman

July 2003

NM|

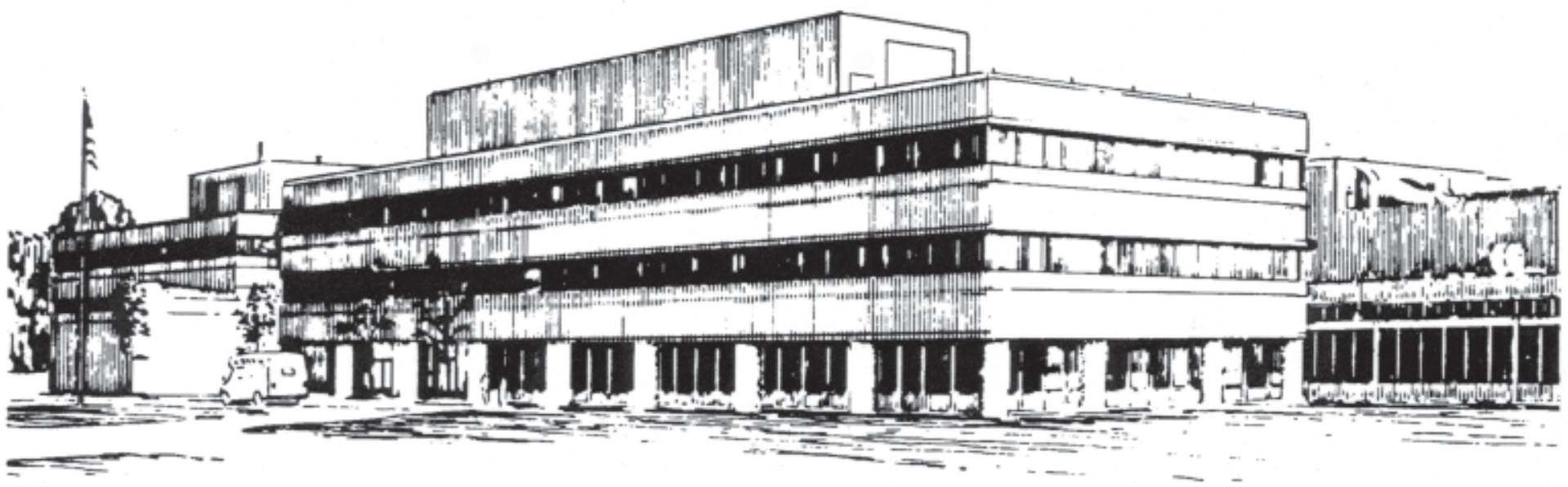

PRINCETON PLASMA PHYSICS LABORATORY PRINCETON UNIVERSITY, PRINCETON, NEW JERSEY 


\section{PPPL Reports Disclaimer}

This report was prepared as an account of work sponsored by an agency of the United States Government. Neither the United States Government nor any agency thereof, nor any of their employees, makes any warranty, express or implied, or assumes any legal liability or responsibility for the accuracy, completeness, or usefulness of any information, apparatus, product, or process disclosed, or represents that its use would not infringe privately owned rights. Reference herein to any specific commercial product, process, or service by trade name, trademark, manufacturer, or otherwise, does not necessarily constitute or imply its endorsement, recommendation, or favoring by the United States Government or any agency thereof. The views and opinions of authors expressed herein do not necessarily state or reflect those of the United States Government or any agency thereof.

\section{Availability}

This report is posted on the U.S. Department of Energy's Princeton Plasma Physics Laboratory Publications and Reports web site in Fiscal Year 2003. The home page for PPPL Reports and Publications is: http://www.pppl.gov/pub_report/

DOE and DOE Contractors can obtain copies of this report from:

U.S. Department of Energy

Office of Scientific and Technical Information

DOE Technical Information Services (DTIS)

P.O. Box 62

Oak Ridge, TN 37831

Telephone: (865) 576-8401

Fax: (865) 576-5728

Email: reports@adonis.osti.gov

This report is available to the general public from:

National Technical Information Service

U.S. Department of Commerce

5285 Port Royal Road

Springfield, VA 22161

Telephone: $1-800-553-6847$ or

(703) $605-6000$

Fax: (703) 321-8547

Internet: http://www.ntis.gov/ordering.htm 


\title{
Circulation in a Short Cylindrical Couette System
}

\author{
Akira Kageyama* \\ Earth Simulator Center, Japan Marine Science and \\ Technology Center, Yokohama 236-0001, Japan \\ Hantao $\mathrm{Ji}^{\dagger}$ \\ Princeton Plasma Physics Laboratory, Princeton, NJ 08543 \\ Jeremy Goodman \\ Princeton University Observatory, Princeton, NJ 08544
}

(Dated: June 17, 2003)

\begin{abstract}
In preparation for an experimental study of magnetorotational instability (MRI) in liquid metal, we explore Couette flows having height comparable to the gap between cylinders, centrifugally stable rotation, and high Reynolds number. Experiments in water are compared with numerical simulations. The flow is very different from that of an ideal, infinitely long Couette system. Simulations show that endcaps corotating with the outer cylinder drive a strong poloidal circulation that redistributes angular momentum. Predicted toroidal flow profiles agree well with experimental measurements. Spin-down times scale with Reynolds number as expected for laminar Ekman circulation; extrapolation from two-dimensional simulations at $R e \leq 3200$ agrees remarkably well with experiment at $R e \sim 10^{6}$. This suggests that turbulence does not dominate the effective viscosity. Further detailed numerical studies reveal a strong radially inward flow near both endcaps. After turning vertically along the inner cylinder, these flows converge at the midplane and depart the boundary in a radial jet. To minimize this circulation in the MRI experiment, endcaps consisting of multiple, differentially rotating rings are proposed. Simulations predict that an adequate approximation to the ideal Couette profile can be obtained with a few rings.
\end{abstract}

*Electronic address: kage@jamstec.go.jp

${ }^{\dagger}$ Electronic address: hji@pppl.gov

${ }^{\ddagger}$ Electronic address: jeremy@astro.princeton.edu 


\section{INTRODUCTION}

Laboratory experiments using liquid metal have made important tests of magnetohydrodynamic (MHD) theories and processes. Examples include laboratory demonstration of the Alfvén wave[1], magnetofluid convection[2], and dynamo action[3]. Recently, a new kind of liquid-metal MHD experiment, motivated by astrophysics, has been proposed by the authors[4, 5] to study magnetorotational instability (MRI), which is believed to dominate the transport of angular momentum in electrically conducting accretion disks. Liquid gallium will be used in a Couette flow between cylinders of radii $r_{1}<r_{2}$ and angular velocities $0<\Omega_{2}<\Omega_{1}$ but $r_{2}^{2} \Omega_{2}>r_{1}^{2} \Omega_{1}$ so that the flow will be stable against conventional Taylor-Couette instabilities (TCI).

Both TCI and MRI are governed by the radial profile of toroidal velocity, $v_{\varphi}(r)$. In an inviscid fluid, TCI occurs wherever the specific angular momentum, $J \equiv r v_{\varphi}$, decreases outwards, $d J^{2} / d r<0$. If the fluid is also a perfect electrical conductor, MRI occurs when the angular velocity, $\Omega \equiv v_{\varphi} / r$, decreases outwards, $d \Omega^{2} / d r<0$. In this case, MRI can occur in an arbitrarily weak axial magnetic field; the field must at any rate be weak enough so that the transit time of Alfvén waves across the flow is less than $\Omega^{-1}$. The usual situation in accretion disks, which are often excellent conductors, is $\Omega \propto r^{-3 / 2}$ (Kepler's law) so that TCI is stabilized but MRI is not. In a fluid with large resistivity and finite but small viscosity, such as liquid metals, the range of flow parameters unstable to MRI shrinks significantly, while the range unstable to TCI is effectively unchanged. Therefore, laboratory flows must be set up with precision in order to demonstrate MRI while suppressing TCI.

In previous linear stability analyses of gallium Couette flow $[4,5]$, we adopted periodic boundary condition in the vertical (axial) direction, ignoring the effects of the top and bottom interior surfaces of the vessel ("endcaps"). The choice of vertical boundary conditions is probably inconsequential when the height of the flow $(H)$ is much larger than the gap width, as in Taylor's classic experiments[6]. Our experimental volume $\pi H\left(r_{2}^{2}-r_{1}^{2}\right)$ will be limited by the availability of gallium, a far more expensive fluid than water, while the gap must be wide enough so that the magnetic diffusion time is not much shorter than the rotation period. These considerations drive us to an aspect ratio $H /\left(r_{2}-r_{1}\right) \sim O(1)$, in which the endcaps may assume great importance.

We have performed a water experiment and complementary numerical simulations to 
study the effects of the endcaps and, if possible, to find a way to set up a short Couette flow that is unstable to MRI yet stable against TCI. Since the viscosities of the two fluids are similar, standard visualization techniques in water serve to predict the flow structure in the opaque liquid gallium, at least in the absence of magnetic field.

The history of fluid dynamics in short cylindrical Couette systems extends to the 19th century[7], but our experiment is atypical in several respects. Since the pioneering work by Benjamin[8-10], the majority of such work has used a stationary outer cylinder with stationary caps, the main interest being the influence of the short geometry on TCI. In our TCI-stable flows, the outer cylinder must rotate. To allow a wide gap, we use a relatively small radial aspect ratio $\eta=r_{1} / r_{2}=0.256$, so that the commonly used narrow-gap approximation does not apply. Lastly, the rotation rate of the inner cylinder $\left(\Omega_{1}\right)$ is so high that the Reynolds number

$$
R e \equiv \frac{r_{1}\left(r_{2}-r_{1}\right) \Omega_{1}}{\nu} \sim O\left(10^{6}\right)
$$

is orders of magnitude larger than typical Couette flow experiments. In connection with research on the tropospheric jet stream, Dunst performed a water experiment in a short cylindrical annulus with parameters similar to ours[11]. We will comment on this paper in section V.

The outline of the paper is as follows. Sec.II describes the experimental apparatus and most of our experimental results, except for spin-down measurements, which are deferred to Sec.IV. Numerical methods are described in Sec.III, and numerical simulations are presented and compared with experiment in Sec.IV. Sec.V contains a summary of our main results and a discussion of their significance.

\section{WATER EXPERIMENT}

\section{A. Experimental Apparatus}

The experiments are performed in a short circular Couette flow illustrated in Fig. 1. A container, made of transparent acrylic plates and cylinders, is mounted on a stainless steel flange which is driven by a DC motor (1.25 HP, by Creative Industries). The speed is measured by a laser-based tachometer. The inner cylinder, made of aluminum, is inserted through a lip seal from the top and is driven in the direction of the container by an AC motor 
(3 HP, by Lincoln Motors) with a controller. For given speeds of inner and outer cylinders, $\Omega_{1}$ and $\Omega_{2}$, the flow typically requires about $10-30$ seconds to reach a steady state, in which most of the flow measurements are performed.

\section{B. Measurement of Toroidal Flow Profiles}

In order to measure flow profiles as a function of radius and height, small particles with sizes on the order of $1 \mu \mathrm{m}$ made of mica and titanium dioxide ("Sparkle" by Lee S. McDonald, Inc.) are mixed into the working fluid (water). Being small, the particles follow the flow well. A sheet of light of approximate thickness of $0.5 \mathrm{~cm}$ is generated by a horizontal slot in front of a bright halogen light source. An electronically gated intensified-CCD camera (by ITT Corp.) images the flow illuminated by the sheet, whose height is adjustable. Images are saved to a PC using a frame-grabber at a rate of 60 images per second. Particles appear in the images as streaks, whose length indicates flow speed. By combining measurements at many radii and heights, the toroidal flow can be mapped out as a function of $r$ and $z$. The measurements were calibrated, at various heights, by imposing uniform rotation, $\Omega_{1}=\Omega_{2}=150 \mathrm{rpm}$.

The experimental results are shown in Fig. 2 for the case of $\Omega_{1}=2000 \mathrm{rpm}$ and $\Omega_{2}=150$ rpm. There are a few characteristics worth mentioning here. First, the measured velocity is significantly smaller than in an ideal, infinitely long Couette flow having the same $\Omega_{1}$ and $\Omega_{2}$ [Eq. (A1)]. The discrepancy is largest at small radii. Secondly, the velocity must rise sharply from $\sim 3 \mathrm{~m} / \mathrm{s}$ to match the inner cylinder at $\sim 8 \mathrm{~m} / \mathrm{s}$. Unfortunately, diagnostic access to the flow is limited near the inner, outer, and top boundaries. Thirdly, the toroidal velocity decreases with radius except at the locations near the outer edge, whereas it would decrease everywhere in the ideal Couette flow. Fourth, the dependence on $z$ is at most comparable to the experimental errors. This is consistent with Taylor-Proudman theorem [12] which predicts small $z$ variations in a rotating flow with small viscosity.

The observed profile of toroidal flow has unfavorable implications for the proposed MRI experiments. The goal is to set up a flow unstable to MRI while stable to TCI. However, the sharp decrease of $v_{\varphi}$ near the inner cylinder will certainly incite TCI while in the rest of the flow, because $v_{\varphi}$ falls more slowly than intended, will be more resistant to the MRI. As a result, the system as a whole could have a mixture of both instabilities or, even worse, 
only the TCI. The observed deviations from ideal Couette flow are due to the endcaps. They need to be understood and, if possible, to be minimized in order to demonstrate MRI unambiguously. We note that a proposed MRI experiment using sodium [13] should suffer from the same complications since its aspect ratio is also small, viz. $H /\left(r_{2}-r_{1}\right)=2$.

\section{NUMERICAL METHODS}

\section{A. Mathematical Model and Algorithm}

We have developed a 2-dimensional computer code to simulate the water experiment described in the previous section. We use the stream function-vorticity method[14] in cylindrical coordinates $(r, \varphi, z)$, as illustrated in Fig. 3. Assuming axisymmetry, $\partial_{\varphi}\left(v_{r}, v_{\varphi}, v_{z}\right)=0$, and incompressibility, $\nabla \cdot \boldsymbol{v}=0$, we can express the poloidal components of the flow in terms of a stream function, $\psi$ :

$$
\begin{aligned}
& v_{r}=\frac{1}{r} \frac{\partial \psi}{\partial z}, \\
& v_{z}=-\frac{1}{r} \frac{\partial \psi}{\partial r} .
\end{aligned}
$$

The toroidal component of vorticity becomes

$$
\left(\frac{1}{r} \frac{\partial^{2}}{\partial r^{2}}-\frac{1}{r^{2}} \frac{\partial}{\partial r}+\frac{1}{r} \frac{\partial^{2}}{\partial z^{2}}\right) \psi=\omega_{\varphi} .
$$

Since the normal component of velocity vanishes, the boundary conditions for $\psi$ are

$$
\begin{array}{lll}
\psi=0 & \text { at } & r=r_{1}, r_{2}, \\
\psi=0 & \text { at } & z= \pm H / 2 .
\end{array}
$$

The boundary conditions for $v_{\varphi}$ are as follows. At the inner and outer cylindrical walls,

$$
\begin{array}{lll}
v_{\varphi}=r_{1} \Omega_{1} & \text { at } & r=r_{1} . \\
v_{\varphi}=r_{2} \Omega_{2} & \text { at } & r=r_{2} .
\end{array}
$$

Since the top and the bottom endcaps of the vessel rotate with the outer cylinder in our apparatus, the boundary condition there are

$$
v_{\varphi}=\frac{r}{r_{2}} \Omega_{2} \quad \text { at } \quad z= \pm H / 2 .
$$


The no-slip conditions on $v_{r}$ at $z= \pm H / 2$ and on $v_{z}$ at $r=r_{1}, r_{2}$ yield boundary conditions for $\omega_{\varphi}$ via Eqs. (2)-(4):

$$
\begin{array}{ll}
\omega_{\varphi}=\frac{1}{r} \frac{\partial^{2} \psi}{\partial r^{2}} \quad \text { at } r=r_{1}, r_{2}, \\
\omega_{\varphi}=\frac{1}{r} \frac{\partial^{2} \psi}{\partial z^{2}} \quad \text { at } \quad z= \pm H / 2 .
\end{array}
$$

The fundamental variables in our numerical simulation are $v_{\varphi}$ and $\omega_{\varphi}$. Their governing equations are

$$
\begin{aligned}
\frac{\partial \omega_{\varphi}}{\partial t} & =\frac{\partial D_{r}}{\partial z}-\frac{\partial D_{z}}{\partial r}+\nu\left(\nabla^{2}-\frac{1}{r^{2}}\right) \omega_{\varphi} \\
\frac{\partial v_{\varphi}}{\partial t} & =-\left(v_{r} \frac{\partial v_{\varphi}}{\partial r}+v_{z} \frac{\partial v_{\varphi}}{\partial z}+\frac{v_{r} v_{\varphi}}{r}\right)+\nu\left(\nabla^{2}-\frac{1}{r^{2}}\right) v_{\varphi}
\end{aligned}
$$

where

$$
\begin{aligned}
D_{r} & =\frac{J}{r^{2}}\left(\frac{\partial J}{\partial r}\right)+\frac{\omega_{\varphi}}{r}\left(\frac{\partial \psi}{\partial r}\right), \\
D_{z} & =\frac{\omega_{\varphi}}{r}\left(\frac{\partial \psi}{\partial z}\right)+\frac{J}{r^{2}}\left(\frac{\partial J}{\partial z}\right), \\
J & =r v_{\varphi},
\end{aligned}
$$

and

$$
\nabla^{2}=\frac{\partial^{2}}{\partial r^{2}}+\frac{1}{r} \frac{\partial}{\partial r}+\frac{\partial^{2}}{\partial z^{2}}
$$

We use second-order spatial differences on a uniform grid, with typical size $N_{r} \times N_{z}=$ $100 \times 100$, and a fourth-order Runge-Kutta method for the temporal integration, with typical time step $\delta t=7.273 \times 10^{-3}$.

The algorithm for each time step is as follows.

1. Integrate the basic Eqs. (12) and (13) to get new $\omega_{\varphi}(i, k)$ and $v_{\varphi}(i, k)$ in the bulk region $\left(2 \leq i \leq N_{r}-1,2 \leq k \leq N_{z}-1\right)$.

2. Solve Eq. (4) for $\psi$ with the new $\omega_{\varphi}$ as source term and boundary conditions (5)-(6).

3. Set the boundary values of $\omega_{\varphi}$ and $v_{\varphi}$ using Eqs. (7), (8), (10) and (11).

4. Get the auxiliary variables $v_{r}, v_{z}, D_{r}, D_{z}$, and $J$ from Eqs. (2)-(3) \& (14)-(16). 


\section{B. Simulation Method}

The boundary conditions (7), (8), and (9) require a jump of $v_{\varphi}(r)$ at the corners $(r=$ $r_{1}, z= \pm H / 2$ ). A commonly used technique to avoid this singularity is to make a small "buffer region" $r_{1} \leq r \leq r_{1}+\epsilon$, in which $v(r, \pm H / 2)$ varies smoothly[15]. But we found that such a "buffer region" is not necessary in our scheme. Following the standard technique, we first set $\epsilon$ to $10 \%$ of the gap width $r_{2}-r_{1}$, and then gradually reduced it to the radial mesh size, $\Delta r$. In the latter case the angular velocity of the boundary jumps from $\Omega_{1}$ to $\Omega_{2}$ between the innermost two grid points $(i=1,2)$. We confirmed that simulation results are not affected by the size of $\epsilon$. All calculations shown in this paper use $\epsilon=\Delta r$.

All calculations begin with both the boundaries and the fluid at rest. The boundaries (side walls and endcaps) accelerate to their final angular velocities over a short time interval $0 \leq t \leq \tau$. We confirmed through many trials that the final state of the flow does not depend upon $\tau$. All simulations shown here were calculated with $\tau=\left(r_{1}-r_{2}\right) /\left(r_{1} \Omega_{1}\right)$, our unit of time.

\section{RESULTS OF SIMULATIONS}

\section{A. Parameters and units}

The simulations shown in this section use the same dimensions and rotation rates as as the experiments described in Sec. II. The computational units of length, velocity, and time are $\ell=r_{2}-r_{1}, v=r_{1} \Omega_{1}$, and $\ell / v$.

The Reynolds number of the experiment [Eq. (1)] is too high for direct numerical simulation. So we start by simulating very low $R e$ and increase it until we find characteristics of the flow that depend only weakly on $R e$ or follow a clear scaling. The largest simulated $R e$ that we report is 3200 .

\section{B. Low Re flows}

The sudden acceleration of the boundaries at the beginning of the simulation induces a stormy flow inside the vessel. At small values of the final rotation rate, i.e. small $R e$, the fluid quickly relaxes to a laminar steady state. 
Figure 4 shows the toroidal speed, $v_{\varphi}$, for $R e=1$. The maximum speed is achieved at the inner cylinder (on left in Fig. 4), where $v_{\varphi}=1$. In this low Re limit, poloidal flow is almost absent; the maximum value of $v_{r}$ and $v_{z}$ is $7.5 \times 10^{-4}$. Note that the jump in $\Omega$ at the lefthand corners of the boundary does not prevent a smooth solution elsewhere.

Actually, as $R e \rightarrow 0$, the solution for the flow can be found in closed form. If we set $v_{r}=v_{z}=\omega_{\varphi}=0$ in the basic equations (12)-(13), the stationary toroidal flow $v_{\varphi}$ satisfies

$$
\nu\left(\nabla^{2}-\frac{1}{r^{2}}\right) v_{\varphi}=0
$$

with the boundary conditions (7)-(9). Wendl[16] has given the analytical solution of this equation with slightly different boundary conditions, corresponding to $\Omega_{2}=0$. The solution to our problem is given simply by adding a uniformly rotating component $r \Omega_{2}$ to Wendl's solution. The $v_{\varphi}$ profile shown in Fig. 4 is essentially identical to the analytical solution thus constructed. This serves as one benchmark for our code.

\section{High Re flows}

We have seen that poloidal flow is negligible in the low Re regime. As we increase Re, poloidal circulation develops and the toroidal flow changes. Figure 5 shows cross sections in the poloidal $(r, z)$ plane for different Re numbers, from 100 to 3200 . Each panel in Fig. 5 is a snapshot of the nonlinearly saturated state.

Figure 5(a) shows that $v_{\varphi}$ has much the same pattern at $R e=100$ as at $R e=1$ (Fig. 4). In both cases, it is symmetric about the horizontal plane $z=0$ and time independent. The flow becomes unsteady at $R e>400$. The asymmetric profile of $R e=800$ [Fig. $5(\mathrm{~d})$ ] results from unsteady flow. All flows above this Reynolds number fluctuate, with an amplitude that increases with $R e$.

One of the important features shown in Fig. 5 is that the contours of $v_{\varphi}$ tend to be parallel to the rotation axis $(z)$. This is a manifestation of the Taylor-Proudman theorem, viz., that low-frequency horizontal motions tend to be independent of height in an inviscid fluid rotating about a vertical axis[17]. The Taylor-Proudman theorem is usually discussed for an almost rigidly rotating fluid, but as shown in the Appendix, a similar tendency exists in differentially rotating flow provided $d J^{2} / d r>0$.

Another characteristic feature of the $v_{\varphi}$ contours in Fig. 5 is their tendency to concentrate 
towards the inner cylinder (at left) at large $R e$. This shows the development of a boundary layer. Boundary layers also develop on the top and bottom endcaps [see panels (e) and (f)]. Note also the increasingly sharp protrusion of the contours on the inner cylinder near the midplane.

The development of the poloidal $\left(v_{r}, v_{z}\right)$ flow is equally interesting. It is shown in the vector plots of Fig. 6 for the same simulations as in Fig. 5. Vectors appear at every third grid point in $r$ and $z$. Their lengths indicate that the poloidal component becomes stronger at higher $R e$. The arrow lengths are linearly normalized. The amplitude of the unit velocity, that is the rotation speed of the inner cylinder, is indicated by arrows on the top of the panels (a) to (f). A radially outward, jet-like flow is seen near the midplane in the higherRe simulations. It is undoubtedly the counterpart of the spike in the $v_{\varphi}$ contours in Fig. 5. This jet-like flow was not expected before we began our numerical simulations. Its structure will be analyzed later. Here we show the temporal behavior of the poloidal flow, including the jet.

The jet flaps unsteadily. Figure 7 shows the time development of the stream function, $\psi$, for $R e=3200$ over almost half of the period of oscillation of the jet. The period of the oscillation is about 36.95 in the normalized time. Figure $7(\mathrm{a})$ is a snapshot taken at $t=1326.68$, and subsequent panels (b) to (f) are taken with regular 5.82 interval time. Throughout the oscillation, the root of the jet (at $r=r_{1}, z=0$ ) remains fixed while its tip flaps violently up and down. The vector plot in panel (f) of Fig. 6 shows a snapshot of this flapping motion at $t=1294.67$.

\section{Profile of Toroidal Flow}

Figure 8 shows $v_{\varphi}(r)$ for different Reynolds numbers $(200 \leq R e \leq 3200)$ at $z=4 \mathrm{~cm}$. (This is the height that the most reliable experimental data can be obtained.) One can see from this figure that the $v_{\varphi}$ profiles are consistent for the higher $R e$ flows, $R e=800,1600$, and 1600. Note also that the profiles for $R e=1600$ and 3200 are very close, suggesting possible convergence at large $R e$.

In Fig. 9, we have superimposed the experimental data for $v_{\varphi}$ at $z=4 \mathrm{~cm}$ (shown also in Fig. 2) on the corresponding profiles from simulations at $R e=1600$ and 3200 . The agreement is remarkable when one considers that the highest $R e$ achieved in simulations, 
which are in $2 \mathrm{D}$, is a factor of 300 smaller than that of the experiment, which is $3 \mathrm{D}$.

We have seen in Fig. 5 that the high $\operatorname{Re}(>400)$ flows are time dependent. To show the amplitude of the temporal fluctuations, snapshots of $v_{\varphi}$ for $R e=1600$ are superimposed in Fig. 10. (The curves in Fig. 9 are time averages.) We can see that the amplitude is larger in the outer half of the flow $(r>9 \mathrm{~cm})$. This can be explained by the flapping motion of the jet (see Fig. 7).

Both experiment and simulation indicate that the $v_{\varphi}$ profile is concave in the outer half of the flow (Fig. 9), and that $v_{\varphi}(r)$ is an increasing function of radius near the outer cylinder $(12 \mathrm{~cm}<r<15 \mathrm{~cm})$. The fluid in this region rotates almost rigidly, on average, at the angular velocity of the outer cylinder and endcaps $\left(\Omega_{2}\right)$.

Snapshots of the angular momentum $J=r v_{\varphi}$ are shown in Fig. 11 for the same simulation and as in Fig. 10. Note that $d J / d r>0$ for the ideal Couette flow (solid curve) since we aim for stability against the Taylor-Couette mode. An interesting feature of this figure is that the simulated $J$ curve is even flatter than the ideal profile. This is a consequence of the poloidal circulation and jet, which tend to mix angular momentum in the interior of the flow.

\section{E. Jet}

In order to extract essential features of the spatial structure of the flow, it would be convenient if we could suppress its temporal fluctuations, especially the flapping of the jet. For this purpose, we have made a set of simulations with the following symmetries imposed:

$$
\begin{aligned}
& v_{r}(r, z)=v_{r}(r,-z), \\
& v_{\varphi}(r, z)=v_{\varphi}(r,-z), \\
& v_{z}(r, z)=-v_{z}(r,-z),
\end{aligned}
$$

Fig. 12 shows the result when $R e=1600$. One can perhaps interpret this state as an average of the flow over one period of the jet's flapping motion.

The panel (a) in Fig. 12 clearly shows the structure of the poloidal circulation. It consists of three main parts; (i) inward flow in boundary layers at the endcaps; (ii) axial flow towards the midplane on the inner cylinder; and (iii) an outward jet centered on the midplane.

The boundary layer on the inner cylinder has a characteristic triangular shape [panel (b) 
in Fig. 12]. The contours are "squeezed" by the inward boundary-layer flows near the top and the bottom caps, while, the tip of the triangle is "pulled" by the outward jet flow.

The contour lines of $v_{\varphi}$ and $\Omega$ [Fig. 12(b) and (c)] suggest that the flow attempts to obey the Taylor-Proudman theorem outside of the boundary layers and the jet. Since the latter regions are thin, especially at high $R e$, viscous forces becomes important there and the Taylor-Proudman theorem does not hold.

The contour lines of $\Omega$, Fig. 12(c), show that the the outer half of the fluid rotates approximately at the angular velocity of the outer cylinder and endcaps, as we have already seen in Figs. 10 and 11. We have also seen that the angular momentum $J$ tends to be uniform in the interior due to the poloidal circulation, as is is clearly shown in by the central void in the contour plot of $J$ in Fig. 12(d).

The structures of the toroidal and poloidal flows are summarized schematically in Fig. 13.

One interesting but unexpected finding of these simulations is the existence of the jet. Its characteristics are summarized as follows: (1) The jet becomes thinner with increasing $R e$, and its width is similar to that of the boundary layers on the caps; and (2) the jet is steady and symmetric at low Re but flaps vertically above a critical Reynolds number between 400 and 800 .

Experimentally, it is not straightforward to confirm the existence of a jet-like radial flow directly. Since the maximum radial velocity in the simulation is only a few percent of the large toroidal flow, the streaks on the camera images would rotate only a few degrees, too little to be resolved by our measurements. At lower rotation speeds, we were able visually to follow relatively large and neutrally buoyant particles in the water. We observed rather rapid outward motions at the midplane after the particles were "sucked" into the boundary layers at the top or bottom endcaps. This is consistent with the jet-like flows indicated by the simulations.

\section{F. Boundary Layers}

The width of the boundary layers (including the jet) depends on Re. As Fig. 6 indicates, higher $R e$ causes thinner boundary layers. Actually, the inward (negative $v_{r}$ ) flow on the top and bottom boundaries is induced by the same mechanism as classical Ekman circulation: a viscous reduction of $v_{\varphi}$ in the boundary layer, leading to an imbalance between outward 
centrifugal force and the inward pressure gradient. The boundary layers at the endcaps in our system do not have uniform width. As Figs. 12(a), (b), and (d) indicate, these layers are thick near the inner cylinder, reaching roughly $10 \%$ of the vessel's height $(H)$. The width monotonically declines with increasing $r$, disappearing into the rigid rotation part of the outer part of the fluid. [See also Fig. 13(b).]

For small departures from rigid rotation, the Ekman layer thickness is $\delta_{E}=\sqrt{\nu / \Omega}$, where $\nu$ is the kinematic viscosity. Our system is very far from rigid rotation, so it is not immediately clear what to substitute for $\Omega$. If one uses the mean frequency $\sqrt{\Omega_{1} \Omega_{2}}$, then for our geometry, $\delta_{E} / H \approx 1.24 R e^{-1 / 2}$, hence $\approx 3 \%$ at $R e=1600$. In fact, we estimate from our simulations that the fractional thickness of the boundary layers is $\sim 10 \%$ at this Reynolds number. For small departures from a differentially rotating state, however, we believe that it is more appropriate to scale $\delta_{E}$ with the epicyclic frequency,

$$
\kappa=\left(\frac{1}{r^{3}} \frac{\partial J^{2}}{\partial r}\right)^{1 / 2} .
$$

This is the maximum frequency of small axisymmetric motions (inertial oscillations) in the inviscid interior of the fluid, so it represents the inertial forces that must be balanced by viscous ones in order to drive a radial flow along the boundaries. Since $\kappa$ reduces to $2 \Omega$ for rigid rotation, we take $\delta_{E}=\sqrt{2 \nu / \bar{\kappa}}$. A characteristic value for $\kappa$ is

$$
\bar{\kappa}=2\left(\frac{r_{2}^{4} \Omega_{2}^{2}-r_{1}^{4} \Omega_{1}^{2}}{r_{2}^{4}-r_{1}^{4}}\right)^{1 / 2}
$$

This leads to $\delta_{E} / H=3.39 R e^{-1 / 2}$, or $\sim 8.5 \%$ at $R e=1600$, which is about three times larger than the previous estimate and closer to the results of the simulations.

A prediction of the latter scaling is that the Ekman-layer thickness should increase along a sequence in which $\left(r_{2}^{2} \Omega_{2}\right) /\left(r_{1}^{2} \Omega_{1}\right)$ approaches unity (from above) while the mean rotation $\sqrt{\Omega_{2} \Omega_{1}}$ is constant.

\section{G. Flow in Shorter Cylinder}

The numerical simulations presented so far have been performed for cylinders of height $H=10 \mathrm{~cm}$, hence about the same as the width of the gap $\left(r_{2}-r_{1}=11.1 \mathrm{~cm}\right)$, as in our

laboratory experiment. In order to elucidate the effects of the top and bottom endcaps on the fluid motion, we have also performed numerical experiments for shorter heights: $H=8,6,2$, 
and $1 \mathrm{~cm}$. Figure 14 shows the corresponding flow profiles after nonlinear saturation. The Reynolds number, which is based on the cylinder radii rather than $H$ [Eq. (1)] is 1600 in all cases. Compare these with Fig. 5(e).

In the case of the shortest height $(H=1 \mathrm{~cm})$, most of the fluid rotates at the angular velocity of the endcaps. This is not surprising, since in the limit of infinitesimal height, the fluid would "adhere" to the endcaps. The more rapidly rotating inner cylinder influences the flow over a radial distance comparable to $H$. Comparing the panels of Fig. 14, we see that the domain of rigid rotation shrinks as $H$ grows, but it still exists when $H=10 \mathrm{~cm}$, as summarized in the schematic Fig. 13(b). The existence of such a region in a short Couette flow has also been reported by Dunst[11].

\section{H. Spin Down}

To better quantify the effects of circulation, a series of experiments and simulations have been performed to study the transient flow when both cylinders (and endcaps) are suddenly stopped. The rate of spin down reflects the efficiency with which the circulation transports angular momentum and the viscous coupling to the walls. In the experiments, starting from steady rotation both cylinders were braked to a complete stop within about one second. The flow speed in a small volume was measured against time, as shown in Fig. 15.

A simple exponential fit to the measured data is not appropriate because the spin-down time $\tau$ itself depends on angular velocity:

$$
\tau=\frac{H}{2 \delta_{E} \bar{\Omega}}=\frac{H}{2 \sqrt{\nu \bar{\Omega}}},
$$

where the factor 2 comes from the fact that the circulation has 2 cells and the Ekman layer thickness $\delta_{E}$ is taken to be $\sqrt{\nu / \bar{\Omega}}(\bar{\Omega}$ is an averaged angular velocity). Thus, we have

$$
\frac{d \bar{\Omega}}{d t}=-\frac{\bar{\Omega}}{\tau} \propto-\bar{\Omega}^{3 / 2},
$$

which leads to

$$
\bar{\Omega}(t)=\frac{\bar{\Omega}\left(t_{0}\right)}{\left(1+\frac{t-t_{0}}{\tau}\right)^{2}} .
$$

The measurements are fit to Eq.(23) where the steady state angular velocity $\bar{\Omega}\left(t_{0}\right)$ is known while $t_{0}$ and $\tau$ are fitting parameters. The fitted line is shown by the dashed line in Fig. 15 and the spin down time, $\tau=11.2 \pm 0.9 \mathrm{sec}$, is obtained. 
Spin down has also been simulated by imposing a sudden stop of all boundaries. Figure 16 shows results for toroidal velocity in the same volume as in the experiment for $R e=3200$. Again, the spin down time is obtained by fitting Eq.(23), with the result $\tau=0.82 \mathrm{sec}$. Figure 17 displays $\tau$ determined similarly for a series of simulations at different $R e$. The trend is well fit by a power law (dashed line),

$$
\tau=0.012 R e^{0.53} \mathrm{sec} .
$$

For the purposes of Fig. 17 and Eq. (24), we fix $\Omega_{1}=2000 \mathrm{rpm}$ and $\Omega_{2}=150 \mathrm{rpm}$ (as in the experiment) and imagine that the Reynolds number of the simulations is controlled by varying the viscosity. If the viscosity is fixed and the rotation rates vary (as would be more convenient in an experiment) then the spindown time scales as $R e^{-0.47}$. The power law (24) agrees excellently with the simple estimate given by Eq.(21),

$$
\tau=\frac{H}{2 \sqrt{\nu \bar{\Omega}}}=\frac{H}{2 \sqrt{r_{1}\left(r_{2}-r_{1}\right) \Omega_{1} \bar{\Omega} R e^{-1}}}=0.011 R e^{1 / 2} \mathrm{sec} .
$$

[For comparison, Eq. (A9) predicts $t_{E}=0.0095 R e^{1 / 2}$ sec.] The experimental point, which was not included in the fit, is rather close to the extrapolation of Eq. (24): $11.2 \pm 0.9 \mathrm{sec}$ observed vs. $17 \pm 0.9 \mathrm{sec}$ predicted, using $\nu=0.01 \mathrm{~cm}^{2} \mathrm{sec}^{-1}$ for water.

\section{SUMMARY AND DISCUSSION}

Motivated by our proposed magnetohydrodynamic experiment $[4,5]$, we have studied a short, wide-gap, hydrodynamic Couette flow by experiment and numerical simulation. A challenge of the gallium experiment will be to set up an initial rotation profile that is stable to the Taylor-Couette instability (TCI) while unstable to the magnetorotational instability (MRI) when an appropriate magnetic field is applied. It is found experimentally that the toroidal flow $v_{\varphi}(r)$ is significantly different from that in an infinitely long circular Couette system. In order to understand the underlying physics, numerical simulations have been performed using the same boundary conditions as in the experiment. The measured profile of $v_{\varphi}$ is successfully reproduced by the simulations, which show a strong poloidal circulation driven by boundary layers at the endcaps. Furthermore, excellent agreement between experiment and simulation is found for the spin-down time when the Reynolds number is scaled. 
These agreements are rather remarkable considering that there is a factor of 300 between the Reynolds numbers of the simulations and of the experiments, and that the simulations are performed in two dimensions, while the experiments are three-dimensional. The suggestion is that the essential dynamics of the system do not change when $R e$ is raised from 3200 to about $10^{6}$.

Is it plausible that the the boundary layer remains laminar up to the experimental Reynolds number $R e=8.8 \times 10^{5}$ ? Perhaps: nonrotating flow over a flat plate remains laminar below $R e_{\text {crit }} \approx 5 \times 10^{5}$; perhaps more relevantly for our experiment, $R e_{\text {crit }} \approx 3 \times 10^{5}$ for a disk spinning freely in an extended, nonrotating fluid, where $R e \equiv \Omega R^{2} / \nu$ based on the angular velocity and radius of the disk [18]. For fully turbulent boundary layers, the stress $(\sigma=$ lateral force per unit area) exerted on the boundary is parametrized by a friction coefficient $C_{f} \equiv \sigma / \frac{1}{2} \rho V_{\infty}^{2}$, where $V_{\infty}$ is the relative velocity of the fluid well outside the boundary layer. It is known that $C_{f}$ varies slowly with $R e$ when $R e$ is large. In flow over smooth surfaces at $R e \gg 10^{6}$, for example, von Kárman prescribes $C_{f}=0.455\left(\log _{10} R e\right)^{-2.58}$ [18]. Let us suppose that we can take $C_{f}$ to be constant over the entire boundary (cylinders and endcaps) for the purpose of estimating the spin-down rate. Taking advantage of the fact that the specific angular momentum varies slowly within our steady-state Couette flow and defining $\bar{J} \equiv\left(r_{1}^{2} \Omega_{1}^{2}+r_{2}^{2} \Omega_{2}^{2}\right) / 2$, we estimate that the total torque on the fluid shortly after the cylinders stop is $\Gamma \approx 2 \pi \rho\left(H+r_{2}-r_{1}\right) \bar{J}^{2} C_{f}$. The total angular momentum of the fluid is $L \approx \pi \rho\left(r_{2}^{2}-r_{1}^{2}\right) H \bar{J}$. Thus the spin-down time becomes

$$
\tau_{f} \equiv \frac{L}{\Gamma}=\frac{H\left(r_{2}^{2}-r_{1}^{2}\right)}{\left(r_{2}^{2} \Omega_{2}+r_{1}^{2} \Omega_{1}\right)\left(H+r_{2}-r_{1}\right)} \approx 0.015 C_{f}^{-1} \text { sec. }
$$

This agrees with the observed value of $11 \mathrm{sec}$ for $C_{f}=1.3 \times 10^{-3}$. For comparison, von Kárman's formula predicts $C_{f}\left(10^{6}\right) \approx 4.5 \times 10^{-3}$. Our Reynolds number however, is not far from $R e_{\text {crit }}$, so turbulence may not be fully developed. Indeed, alternative definitions of the Reynolds number fall even closer to the critical value: for example, $\bar{J} / \nu \approx 3.0 \times 10^{5}$.

Detailed analysis of the simulations show that the poloidal circulation consists of two cells. A strong radially inward flow forms near each of the endcaps in a thin boundary layer. After turning into a vertical flow along the inner cylindrical wall, these layers merge at the midplane into a jet-like, radially outward flow to complete the circulation. The existence of such a jet-like feature appears not to have been recognized previously. Dunst performed a water experiment in short cylindrical annulus with similar condition to ours[11]. One set of 
five experiments by Dunst was carried out with rigid endcaps fixed to the outer cylinder. (Other experiments were done with a free upper surface.) Dunst reported the formation of a two-cell pattern as well as a region of rigid-body rotation in the outer part of the fluid. However, there was no description of a jet between the cells. It is possible that the jet was just overlooked. We note that although we have preliminary evidence for a jet-like flow at the midplane between two cells in the experiment, it is difficult to visualize it and to measure its detailed characteristics. We also carried out simulations in which the outer cylinder is stationary, leading presumably to TCI instabilities. It is interesting that the jet does not form in the latter situation, which has been the main focus of experimental effort in short Couette flows.

We have seen that a region of rigid-body rotation occurs in the outer part of the system. This has been explained by the tendency of the flow to "adhere" to the outer cylinder and endcaps. An alternative setup of the apparatus would be to have the caps rotate rigidly with the inner rather than the outer cylinder. In that case, rigid-body rotation would be expected to appear in the inner part of the flow. Actually, such a fluid-dynamical system has been investigated in the literature in connection with hard disk drives for computers[19-23]. It has been shown in these studies that most of the fluid rotates rigidly with the disks.

The poloidal circulation, and especially the jet, found in this study are an interesting phenomenon in rotating fluids. They transport angular momentum efficiently and reduce the free energy available for shear-driven instabilities. Therefore, we will need to minimize this circulation in the MRI experiment.

One idea for reducing the effect of endcaps is to use a tapered section[24]. Another idea is to divide the cap into two parts: the inner one fixed to the inner cylinder, and the outer one fixed to the outer cylinder. Here, we expand on the latter idea. We divide the endcaps into multiple rings that rotate independently (see Fig. 18). The angular velocity of each ring is chosen according to its center radius and the circular Couette flow.

Figure 19 shows the profiles of $v_{\varphi}$ at $R e=1600$ for three choices of the number of rings. The parallel contour lines in the figure indicate that the fluid is in a Taylor-Proudman state. The effect of the endcaps is highly localized in its vicinity and the poloidal circulation is suppressed almost perfectly. Without the circulation, the stationary flow in this differentially rotating system is very close to that of an infinitely long, ideal circular Couette flow. This is confirmed by the $v_{\varphi}$ profile at the midplane when 3 and 5 rings are used (Fig. 20). 
The rings will reduce the relative velocity between the boundary and the interior flow in steady state, to the point where a turbulent boundary is unlikely (see the discussion above). This makes it more likely that the simulations accurately predict the interior flow despite their relatively low Reynolds number.

An optimization process incorporating this idea is underway to design a gallium experiment with maximum controllability of the flow profile, yet with minimum complications to engineering and experimental operations.

\section{APPENDIX A: EKMAN LAYER IN A DIFFERENTIALLY ROTATING FLOW}

Consider a steady axisymmetric flow that departs only slightly from a centrifugally stable Couette profile,

$$
\Omega=a+\frac{b}{r^{2}}, \quad a, b>0 \& \text { constant }
$$

One may linearize the equations of motion (12)-(16) about the basic state (A1). Marking first-order quantities with the prefix $\delta$, we have $\psi \rightarrow \delta \psi, \omega_{\varphi} \rightarrow \delta \omega_{\varphi}$, and $v_{\varphi} \rightarrow r \Omega+\delta v_{\varphi}$. Linearizing Eq. (12) and discarding time derivatives,

$$
2 \Omega \partial_{z} \delta v_{\varphi}+\nu \Delta \omega_{\varphi}=0
$$

where $\Delta \equiv\left(\nabla^{2}-r^{-2}\right)$. Incompressibility implies $\partial_{z} \delta v_{z}=-r^{-1} \partial_{r}\left(r \delta v_{r}\right)$, whence

$$
\partial_{z} \delta \omega_{\varphi}=\partial_{z}\left(\partial_{z} \delta v_{r}-\partial_{r} \delta v_{z}\right)=\Delta \delta v_{r}
$$

so that

$$
2 \Omega \partial_{z}^{2} \delta v_{\varphi}=-\nu \Delta^{2} \delta v_{r}
$$

By similar steps, Eq. (13) yields

$$
\frac{1}{r} \frac{d J}{d r} \delta v_{r}=\nu \Delta v_{\varphi}
$$

For the Couette profile (A1), the coefficient of $\delta v_{r}$ in this last equation is $2 a$, a constant. Eliminating $\delta v_{r}$ between Eqs. (A2)\&(A3) yields

$$
\left(\kappa^{2} \partial_{z}^{2}+\nu^{2} \Delta^{3}\right) \delta v_{\varphi}=0
$$

When viscosity can be neglected and $\kappa^{2}>0$, Eqs. (A2)-(A4) imply that small, low-frequency $(\ll \kappa)$ motions are independent of $z$ : this is the Proudman theorem. 
We apply these equations to the boundary layer at one of the endcaps, which rotates differentially (as might be achieved by dividing it into many rings, see $\S \mathrm{V}$ ) with a slightly different profile $\Omega_{\text {cap }}(r)$ from that of the fluid in the interior, Eq. (A1). Vertical derivatives are much larger than radial ones in the boundary layer, so Eq. (A4) implies that the boundary-layer behavior is $\delta v_{\varphi} \propto \exp (k z)$ with

$$
k^{4}=-(\kappa / \nu)^{2}, \quad k=( \pm 1 \pm i) \sqrt{\frac{\kappa}{2 \nu}} .
$$

Of these four roots, only the two for which $\delta v_{\varphi}$ decays with distance from the boundary are admissible. To be definite, let us consider the lower endcap, so that $\Re(k)<0$. The Ekman layer thickness is

$$
\delta_{E} \equiv \sqrt{\frac{2 \nu}{\kappa}},
$$

and

$$
\begin{aligned}
\delta v_{\varphi} & =r\left(\Omega_{\text {cap }}-\Omega\right) e^{-z / \delta_{E}} \cos \left(z / \delta_{E}\right), \\
\delta v_{r} & =\frac{\kappa}{a}\left(\Omega_{\text {cap }}-\Omega\right) e^{-z / \delta_{E}} \sin \left(z / \delta_{E}\right)
\end{aligned}
$$

if we take $z=0$ at the endcap rather than the midline of the cylinders. The radial mass flow is

$$
\dot{M}_{E}=2 \pi \rho r \int_{0}^{\infty} \delta v_{r} d z=\pi \rho r^{2}\left(\Omega_{\text {cap }}-\Omega\right) \frac{\kappa}{a} \delta_{E} .
$$

The net torque exerted on the fluid by both endcaps is

$$
\Gamma=2 \int_{r_{1}}^{r_{2}} \dot{M}_{E} \frac{d J}{d r} d r=4 \pi \rho \int_{r_{1}}^{r_{2}} \kappa \delta_{E} r^{3}\left(\Omega_{\text {cap }}-\Omega\right) d r,
$$

and the amount of angular momentum that must be added to the fluid to make its rotation profile agree with the endcaps is

$$
L^{\prime}-L=2 \pi \rho H \int_{r_{1}}^{r_{2}} r^{3}\left(\Omega_{\text {cap }}-\Omega\right) d r .
$$

We may estimate the spin-up or spin-down time as

$$
t_{E}=\frac{L^{\prime}-L}{\Gamma} \approx \frac{H}{\sqrt{8 \nu \bar{\kappa}}} .
$$

Here $\bar{\kappa}$ is a weighted average over radius; if we approximate it by Eq. (20) then Eq. (A9) predicts $t_{E} \approx 9 \mathrm{sec}$ for water with the values of $r_{1}, r_{2}, \Omega_{1}, \Omega_{2}$, and $H$ in Figs. (1) \& (15). The agreement with the measured value $(11.2 \pm 0.9 \mathrm{sec})$ is perhaps better than we deserve in view of the crudeness of the theoretical treatment. In particular, since $\Omega_{\text {cap }}=0$ in the spindown experiment, our linear approximation is not applicable. 


\section{ACKNOWLEDGMENTS}

This work was carried out when one of the authors (A.K.) was staying at Princeton Plasma Physics Laboratory. A.K. thanks to W. Tang for his hospitality. Financial support to A.K. by Japanese Ministry of Education, Culture, Sports, and Technology is gratefully acknowledged. We used NEC SX-5 of National Institute for Fusion Science, Japan for the

numerical simulations. The experimental work was supported by the US Department of Energy. Support has also been received from the National Science foundation under grant AST-0205903. Technical assistance from R. Cutler and experimental contributions from F. Chen and E. Shoshan are gratefully acknowledged.

[1] S. Lundquist, Nature 164, 145 (1949).

[2] Y. Nakagawa, Nature 175, 417 (1955).

[3] A. Gailitis, O. Lielausis, S. Dement'ev, E. Platacis, A. Cifersons, G. Gerbeth, T. Gundrum, F. Stefani, M. Christen, H. Haenel, et al., Phys. Rev. Lett. 84, 4365 (2000).

[4] H. Ji, J. Goodman, and A. Kageyama, Mon. Not. Astron. Soc. 325, L1 (2001).

[5] J. Goodman and H. Ji, J. Fluid Mech. 462, 365 (2002).

[6] G. I. Taylor, Phil. Trans. Roy. Soc. London A 223, 289 (1923).

[7] A. Mallock, Phil. Trans. Roy. Soc. London A 187, 41 (1896).

[8] T. B. Benjamin, Proc. Roy. Soc. London A 359, 1 (1978).

[9] T. B. Benjamin, Proc. Roy. Soc. London A 359, 27 (1978).

[10] T. B. Benjamin and T. Mullin, Proc. Roy. Soc. London A 377, 221 (1981).

[11] M. Dunst, J. Fluid Mech. 55, 301 (1972).

[12] G. Batchelor, An Introduction to Fluid Dynamics (Cambridge University Press, 1967).

[13] K. Noguchi, V. Pariev, S. Colgate, H. Beckley, and J. Nordhaus, Astrophys. J. 575, 1151 (2002).

[14] J. H. Ferziger and M. Peric, Computational Methods for Fluid Dynamics (Springer, 2002), 3rd ed.

[15] T. Mullin, D. Satchwell, and Y. Toya, in Physics of Rotating Fluids, edited by C. Egbers and G. Pfister (Springer, 2000), pp. 3-21. 
[16] M. C. Wendl, Phys. Rev. E 60, 6192 (1999).

[17] S. Chandrasekhar, Hydrodynamic and Hydromagnetic Stability (Dover, 1968).

[18] H. Schlichting, Boundary-Layer Theory (McGraw-Hill, New York, 1979), 7th ed.

[19] S. D. Abrahamson, J. K. Eaton, and D. J. Koga, Phys. Fluids A 1, 241 (1989).

[20] J. A. C. Humphrey, C. A. Schuler, and D. R. Webster, Phys. Fluids 7, 1225 (1995).

[21] R. Kaneko, S. Oguchi, and K. Hoshiya, Review of the Electrical Communication Laboratories, Nippon Telegraph and Telephone Public Corp., Japan 25, 1325 (1977).

[22] E. Lennemann, IBM J. Res. Develop. pp. 480-488 (1974).

[23] C. A. Schuler, W. Usry, B. Weber, J. A. C. Humphrey, and R. Greif, Phys. Fluids A 2, 1760 (1990).

[24] D. S. Cannell, M. A. Dominguez-Lerma, and G. Ahlers, Phys. Rev. Lett. 50, 1365 (1983). 


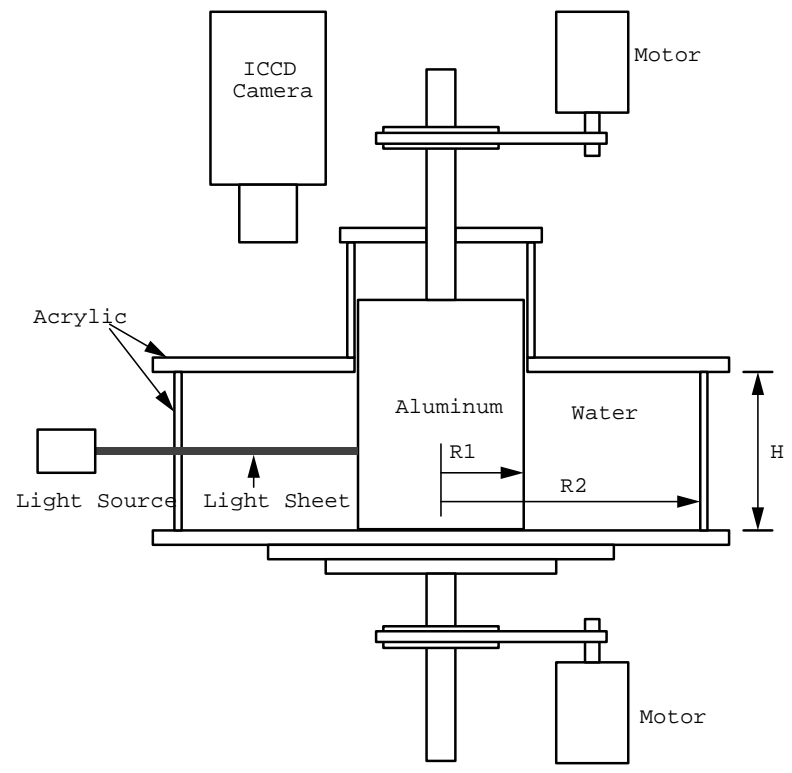

FIG. 1: Schematic of experimental apparatus. Radii of the inner and outer cylinders are $r_{1}=3.8$ $\mathrm{cm}$ and $r_{2}=14.9 \mathrm{~cm}$, respectively. The height is $H=10 \mathrm{~cm}$.

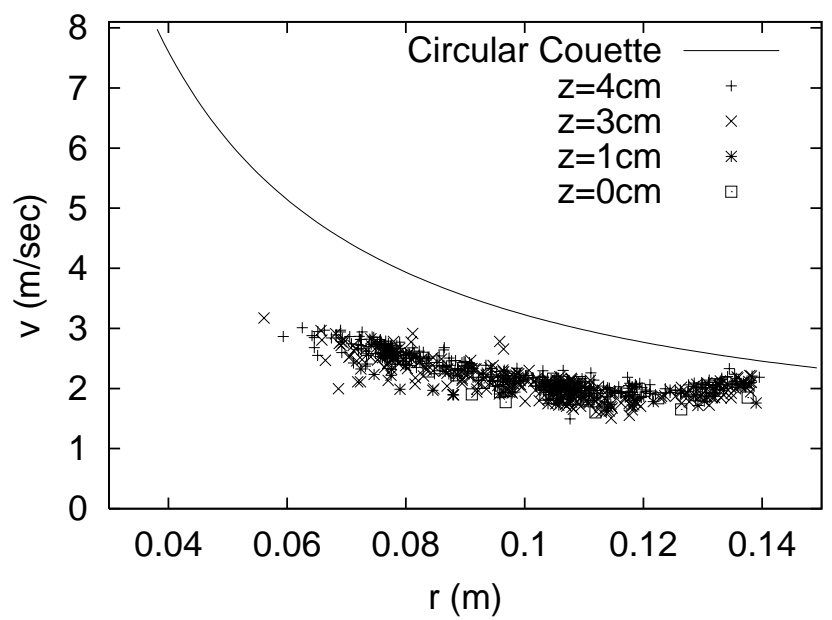

FIG. 2: Measured $v_{\varphi}$ profile at different $z$ when $\Omega_{1}=2000 \mathrm{rpm}$ and $\Omega_{2}=150 \mathrm{rpm}$. 
(a)

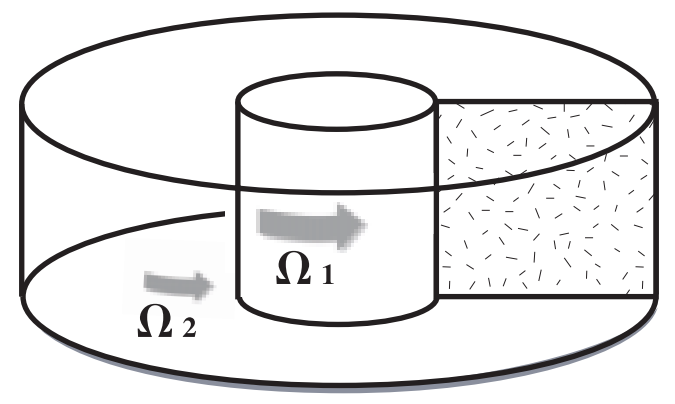

(b)

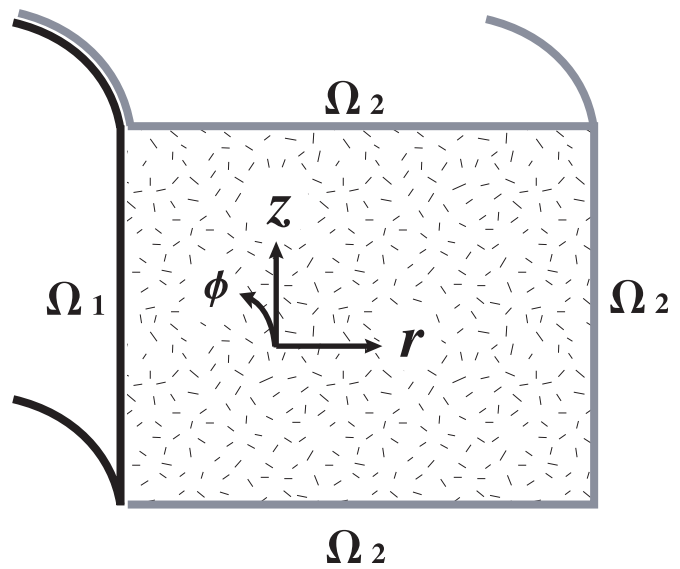

FIG. 3: Illustrations of physical system (a) and simulation system (b).

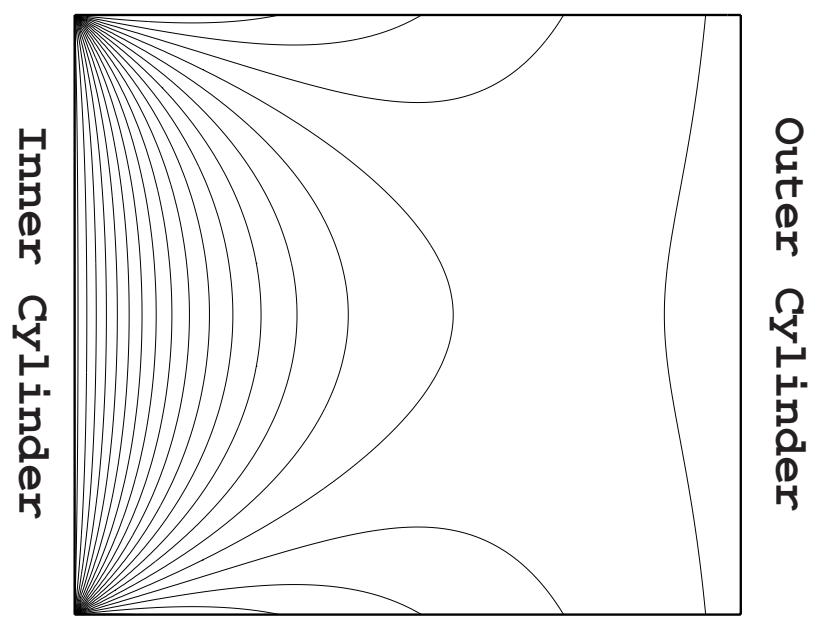

FIG. 4: Simulated $v_{\varphi}$ profile when $R e=1$. 
(a) $\mathrm{Re}=100$

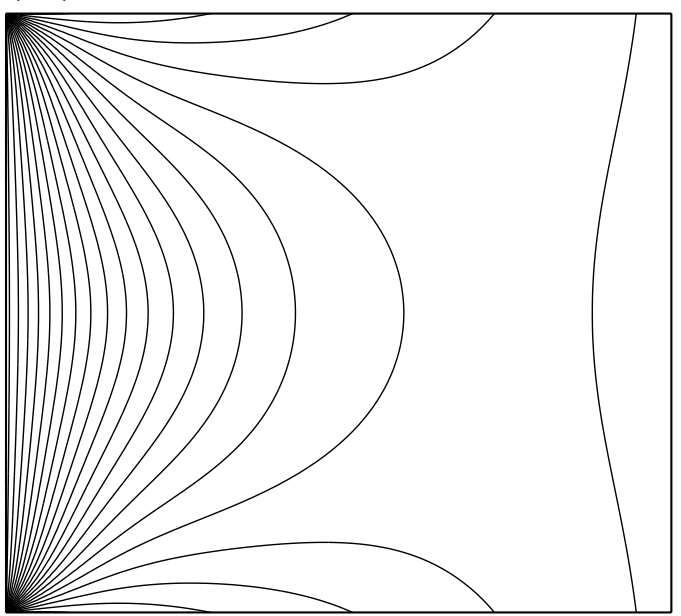

(c) $\mathrm{Re}=400$

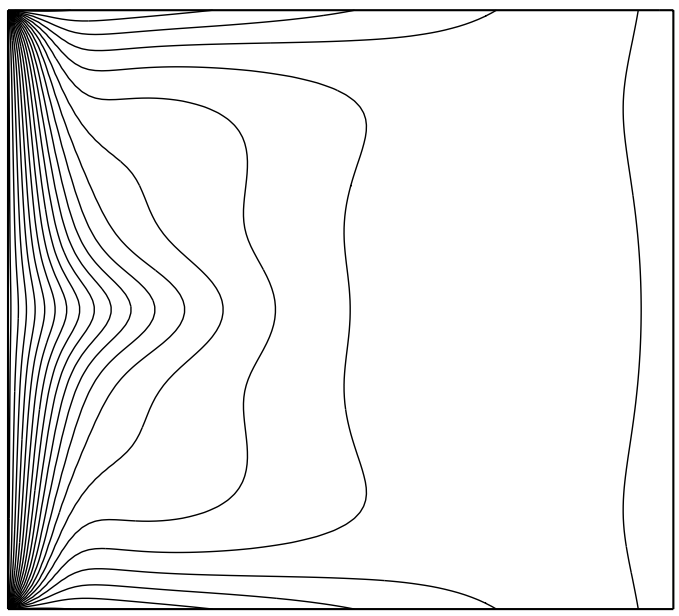

(e) $\mathrm{Re}=1600$

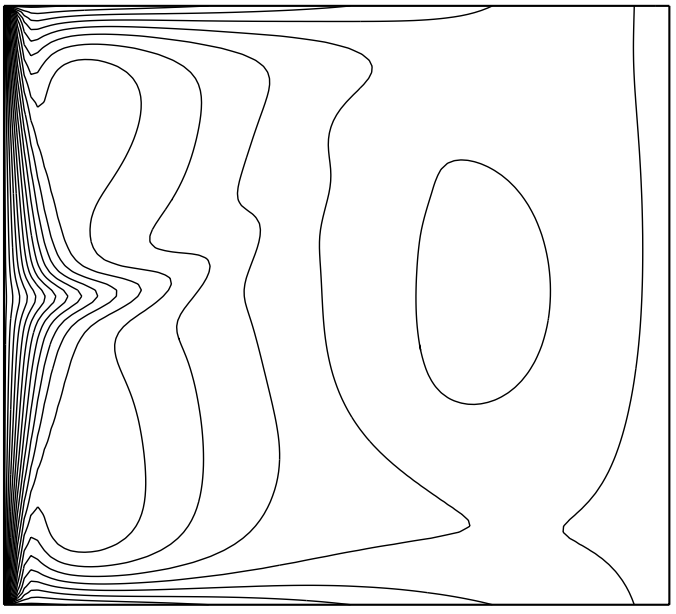

(b) $\quad R e=200$

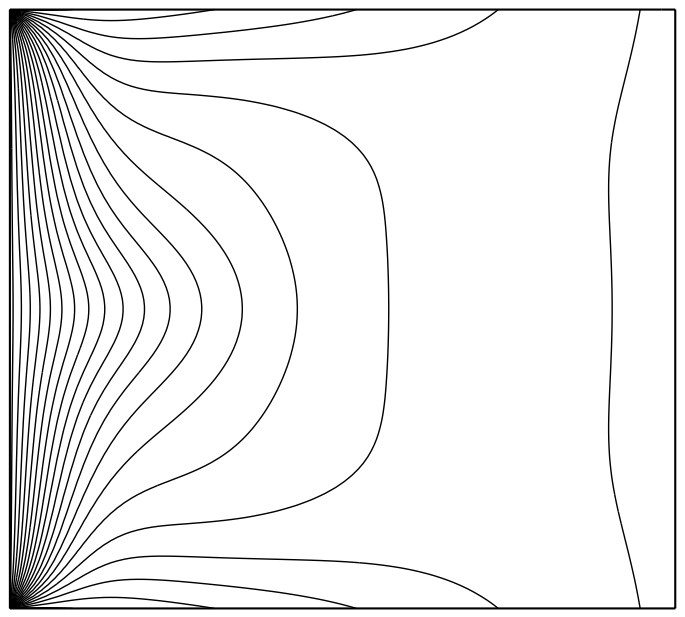

(d) $\mathrm{Re}=800$

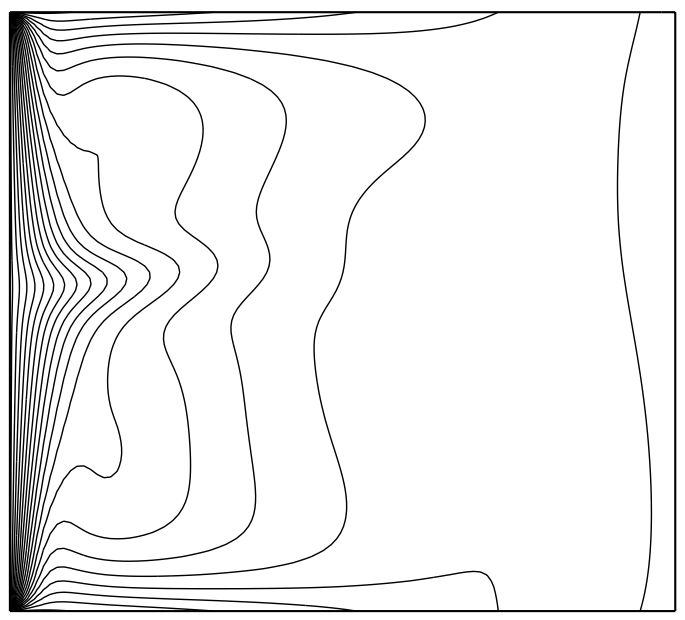

(f) $\mathrm{Re}=3200$

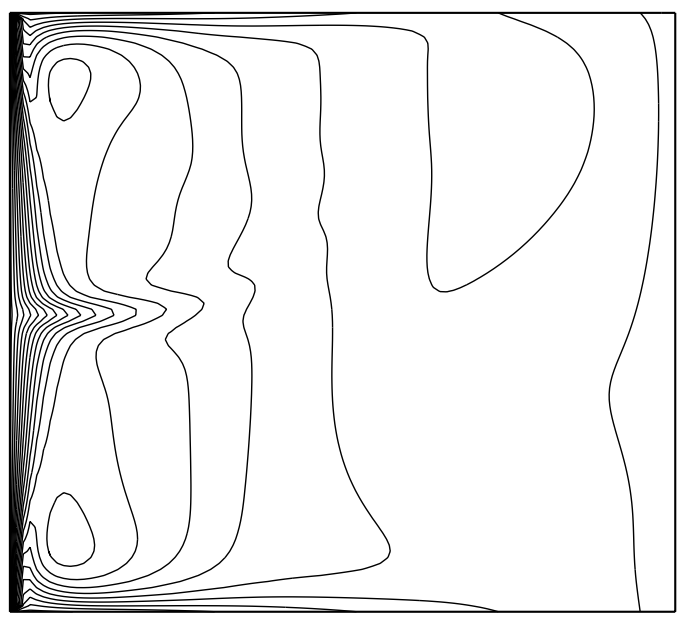

FIG. 5: $V_{\varphi}$ profile.

23 

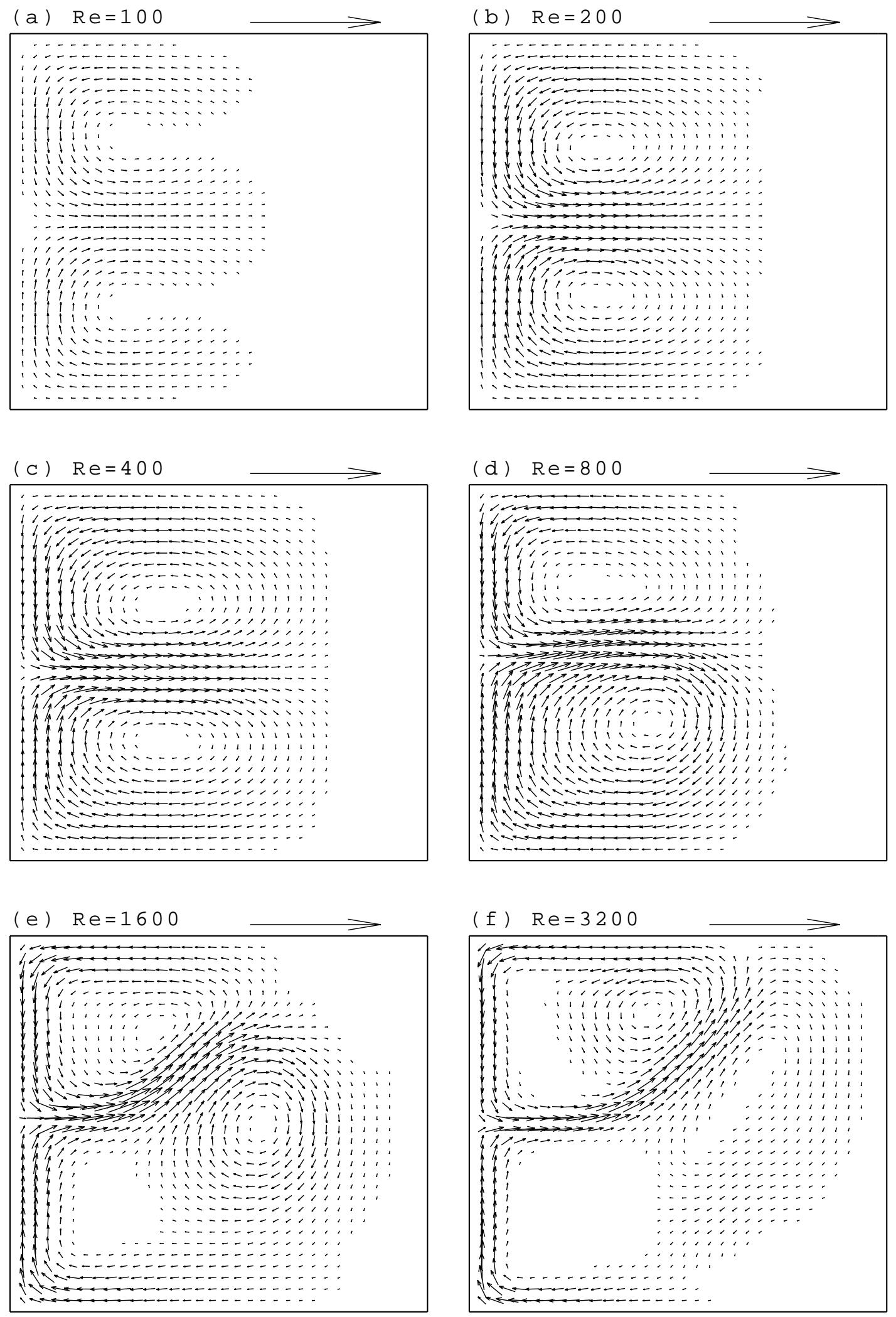

FIG. 6: Poloidal flow profile. 

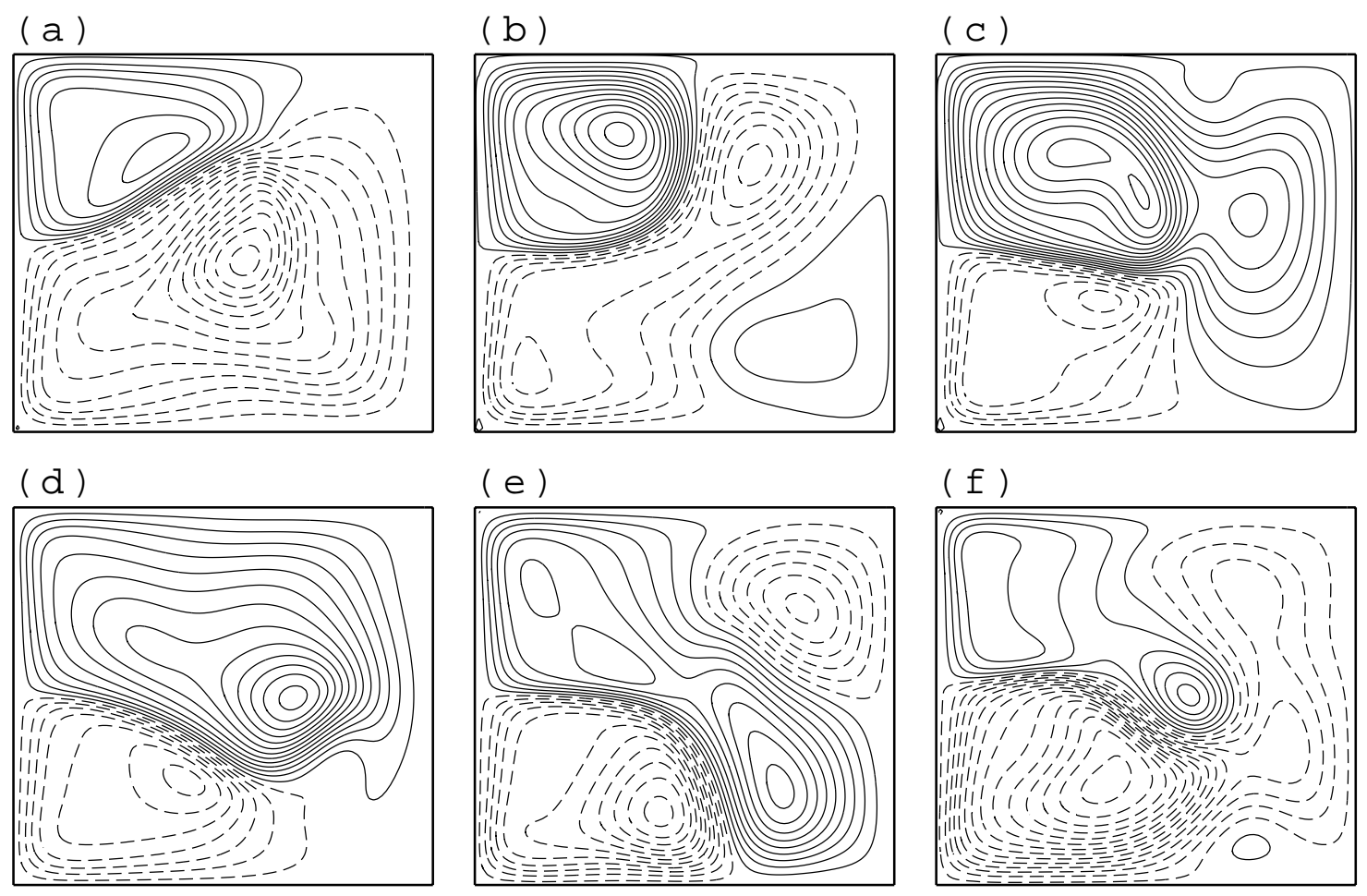

FIG. 7: Contour plots of the stream function in a time sequence showing the oscillatory motion of jet $(R e=3200)$.

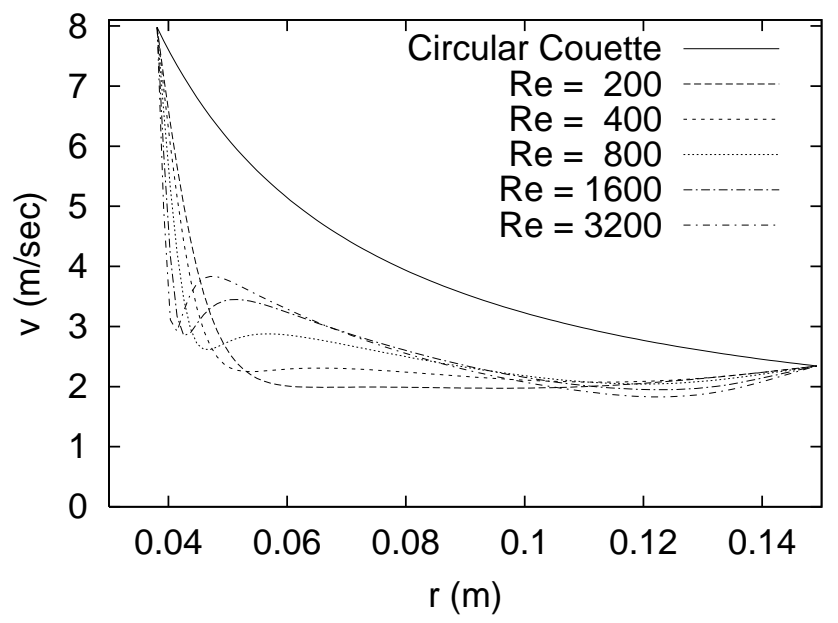

FIG. 8: Simulated $v_{\varphi}$ profile at $z=0$. 


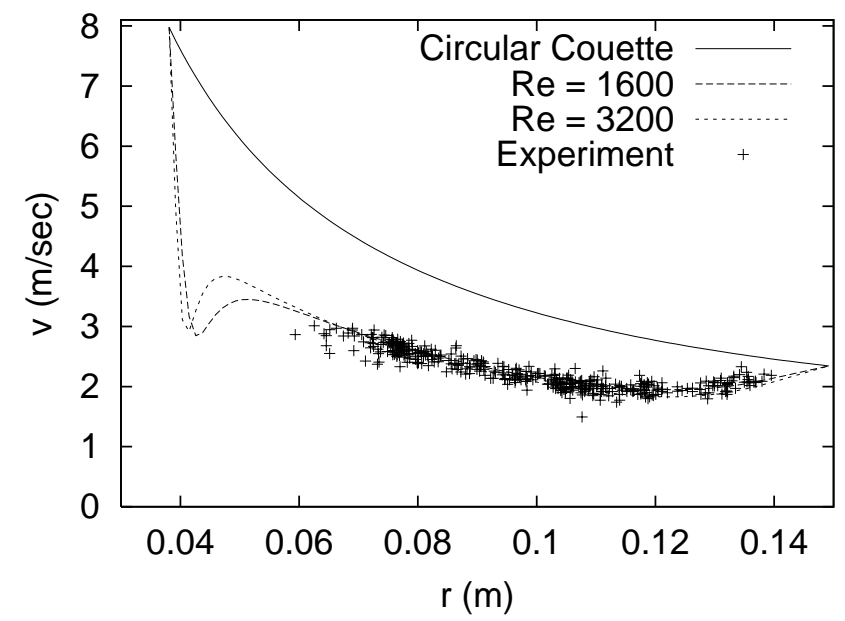

FIG. 9: Comparison of $v_{\varphi}$ profiles obtained by simulation and experiment.

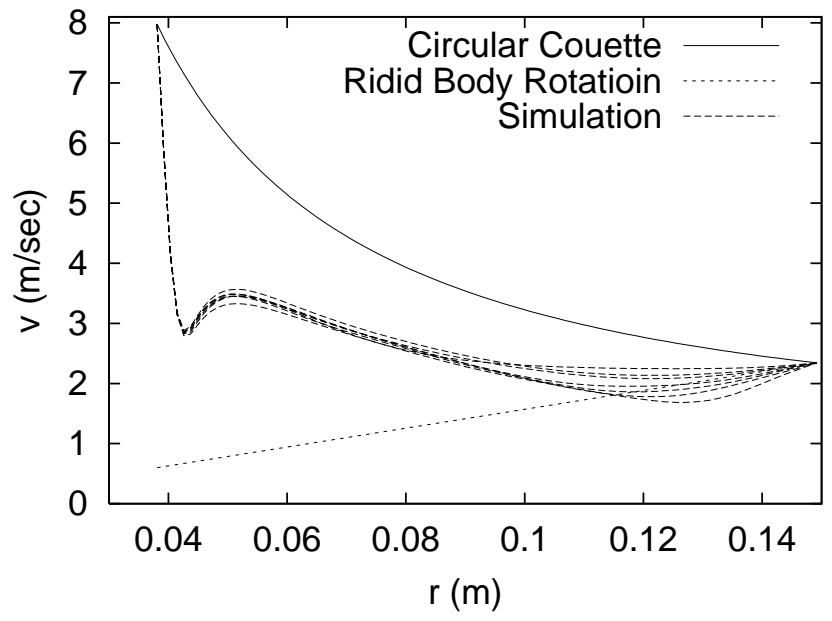

FIG. 10: Time variation of $v_{\varphi}$ profile for $R e=1600$. The rigid rotation profile of the endcaps and outer cylinder shown for comparison. 


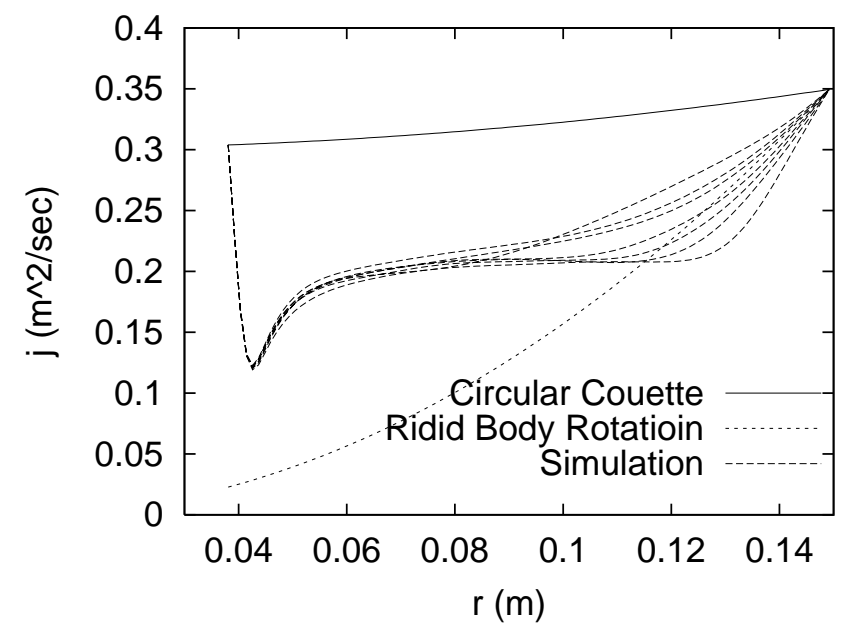

FIG. 11: Time variation of angular momentum $J=r v_{\varphi}$ for $R e=1600$.

(a)

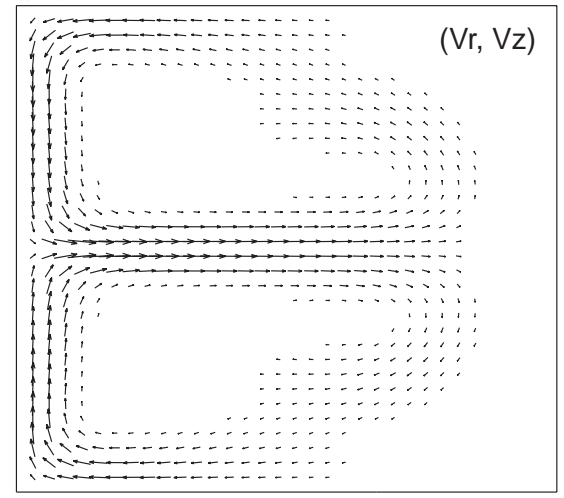

(c)

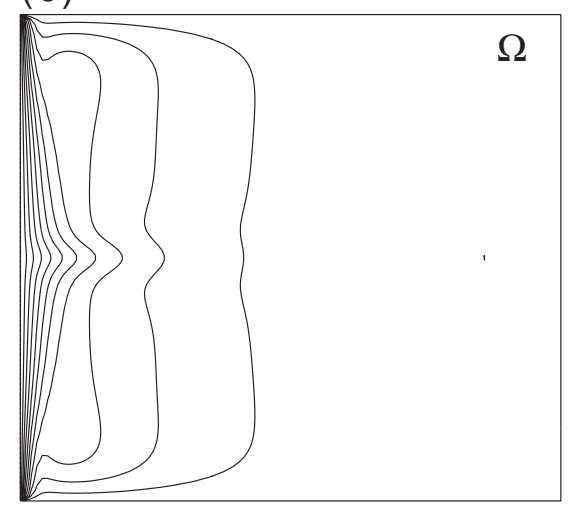

(b)

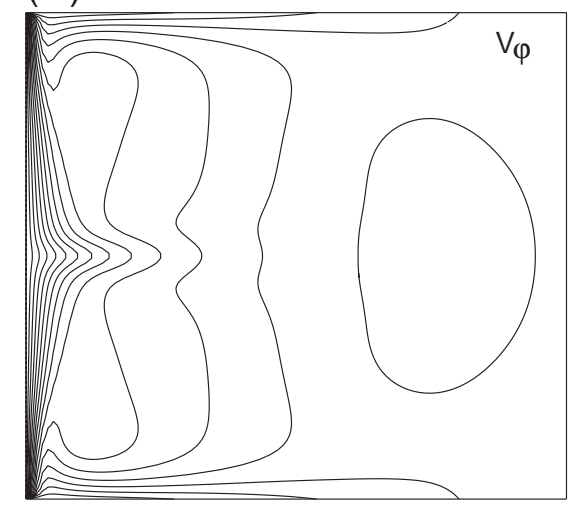

(d)

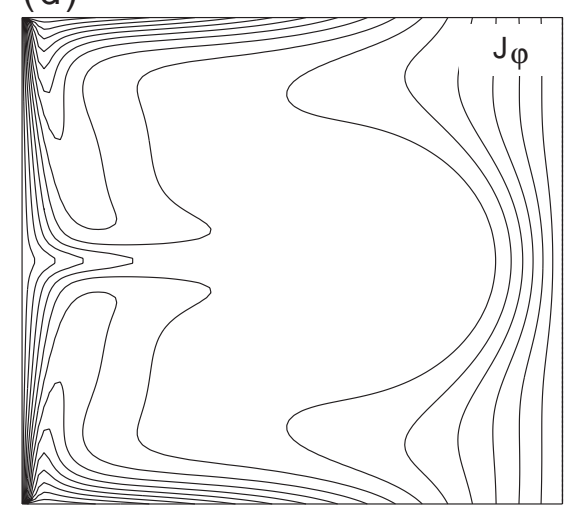

FIG. 12: Flow under enforced symmetry at $R e=1600$ : (a) poloidal flow; (b) contours of toroidal velocity $v_{\varphi} ;$ (c) angular velocity $\Omega$; and (d) angular velocity $J$. 


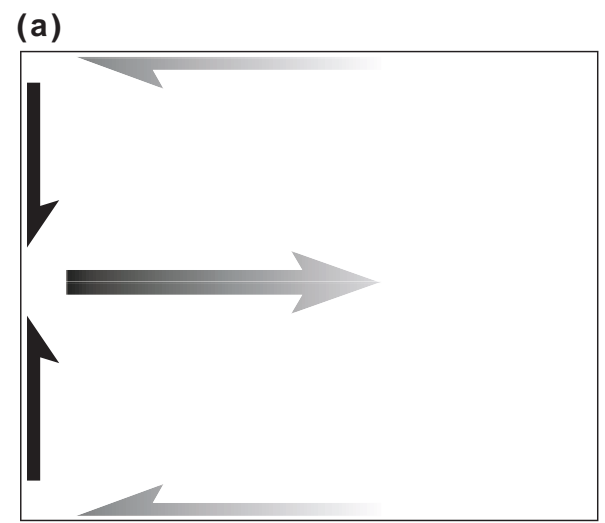

(b)

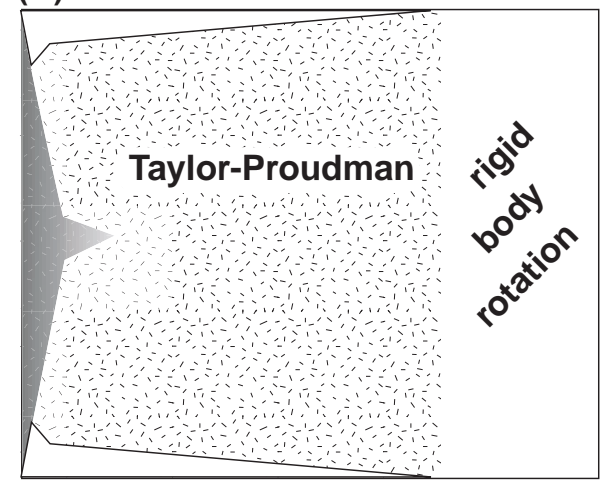

FIG. 13: Schematic flow structure. (a) The Poloidal flow in the boundary layers and the jet. (b) Toroidal flow structure. 

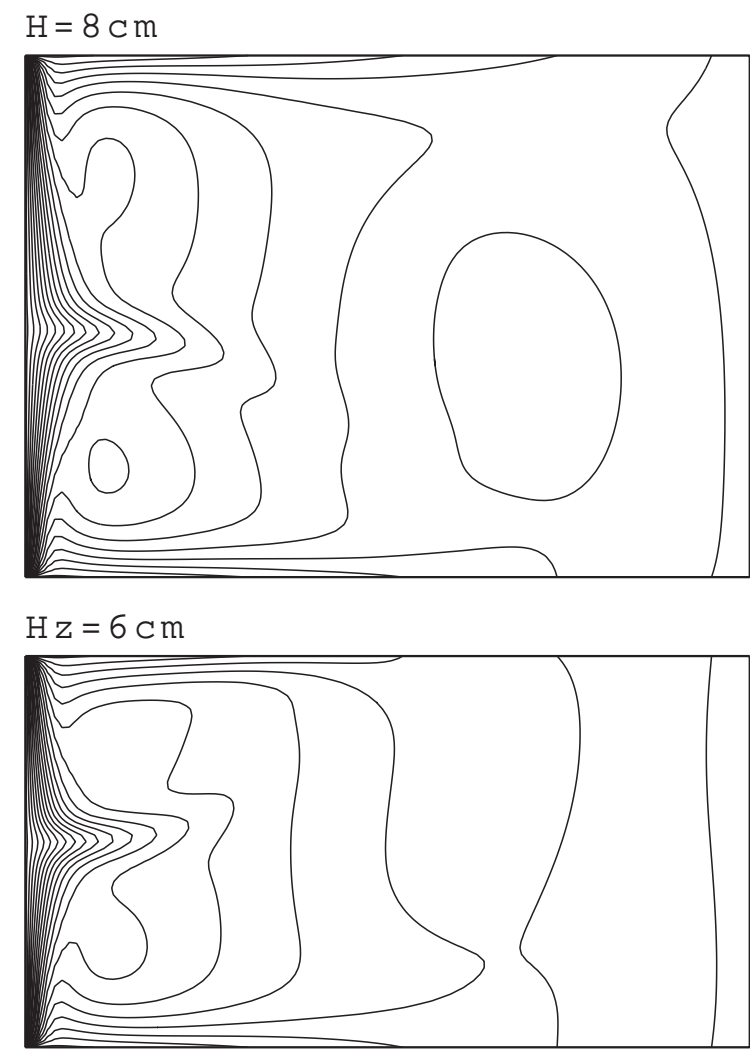

$\mathrm{H} \mathrm{z}=2 \mathrm{Cm}$

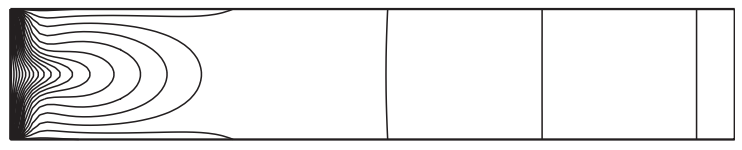

$\mathrm{H} \mathrm{z}=1 \mathrm{~cm}$

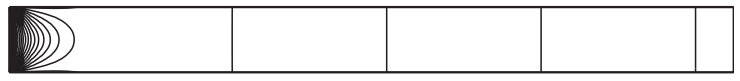

FIG. 14: Profiles of toroidal flow $v_{\varphi}$ for $R e=1600$ for shorter heights: $H=8 \mathrm{~cm}, 6 \mathrm{~cm}, 2 \mathrm{~cm}$, and $1 \mathrm{~cm}$. The corresponding picture for $H=10 \mathrm{~cm}$ is Fig. $6(\mathrm{e})$. 


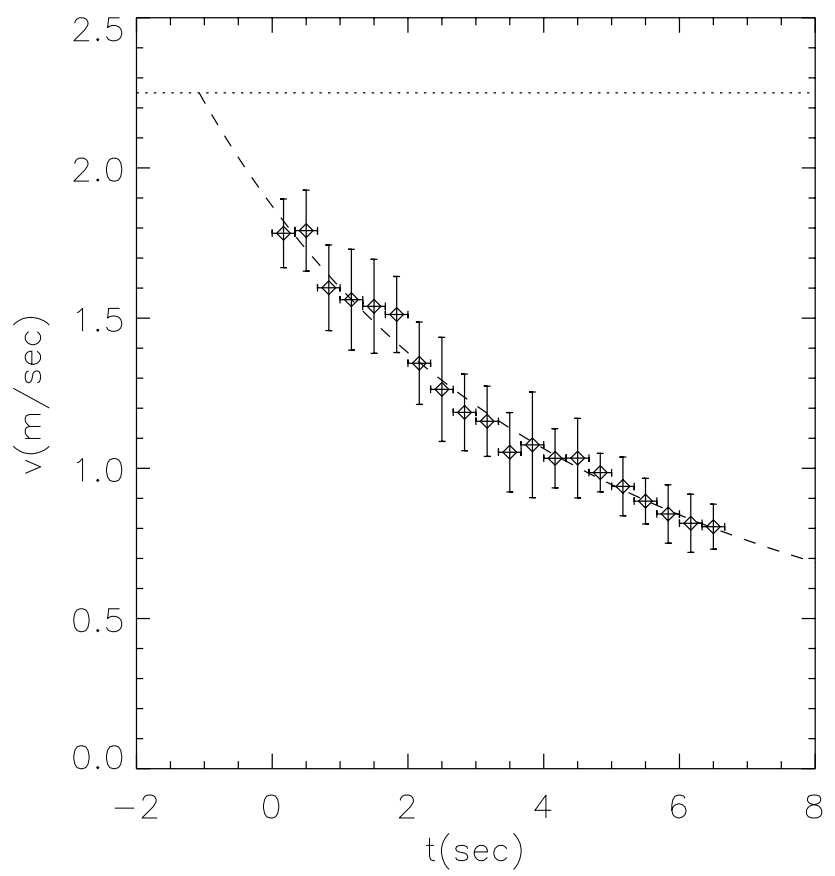

FIG. 15: Experimentally measured toroidal velocity in the region $(r, z) \in(11.5 \pm 1,3 \pm 0.5) \mathrm{cm}$ during spin-down after both cylinders and endcaps are stopped at approximately $t=-1$ sec. Dotted line is toroidal velocity in steady state when $\Omega_{1}=2000 \mathrm{rpm}$ and $\Omega_{2}=150 \mathrm{rpm}$. Dashed line is a fit of the form (23).

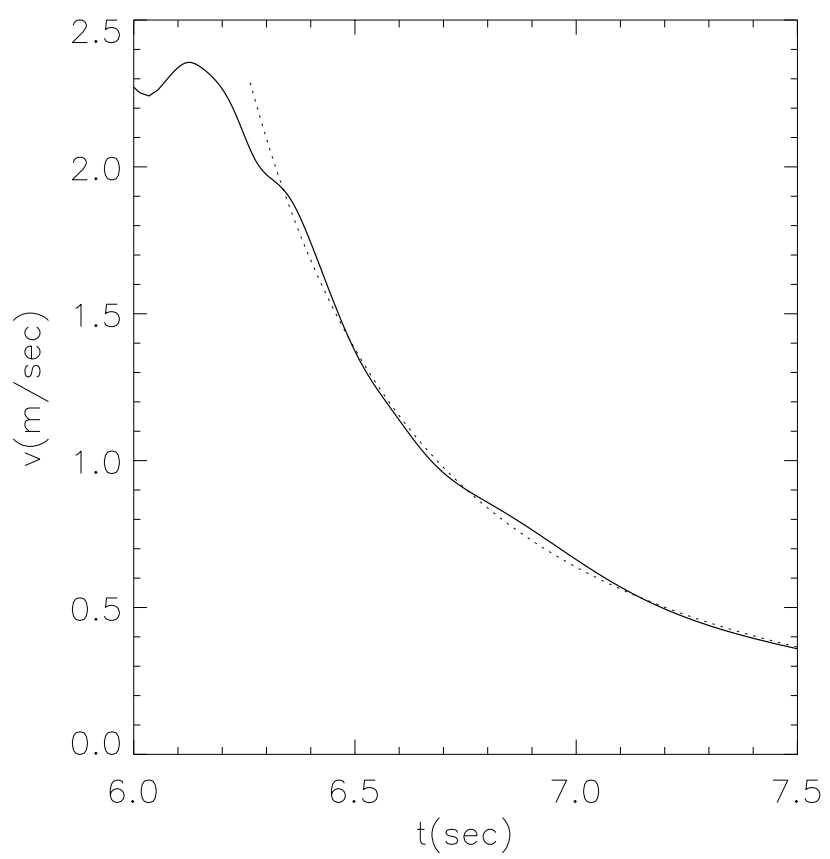

FIG. 16: Like Fig. 15, but for a simulation at initial $R e=3200$. The boundaries are stopped at $t=6.0747 \mathrm{sec}$. 


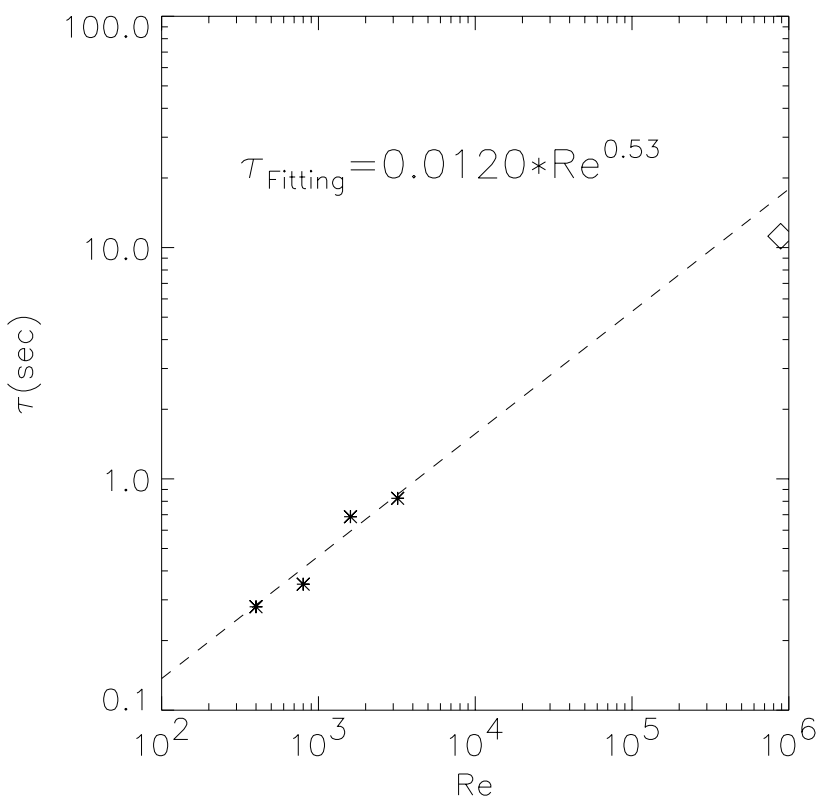

FIG. 17: Spin down time versus $R e$ for simulations (asterisks) and experiment (diamond). Dotted line is a fitted curve to the results from simulation only.

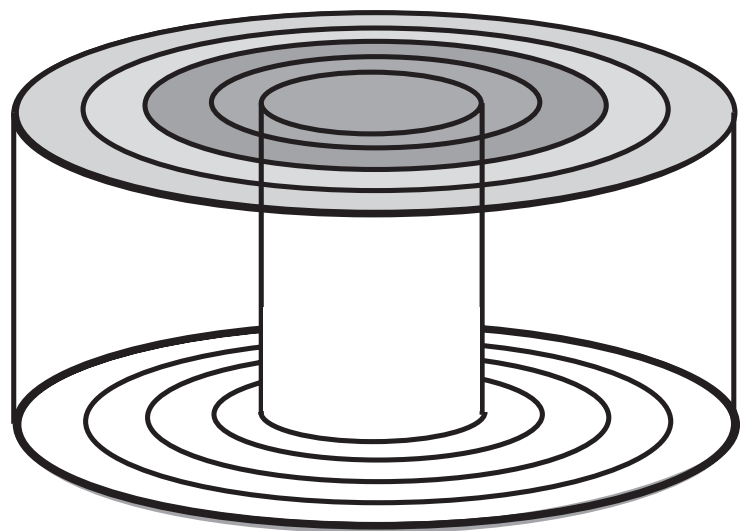

FIG. 18: Experimental setup with independently rotating rings in the endcaps. 

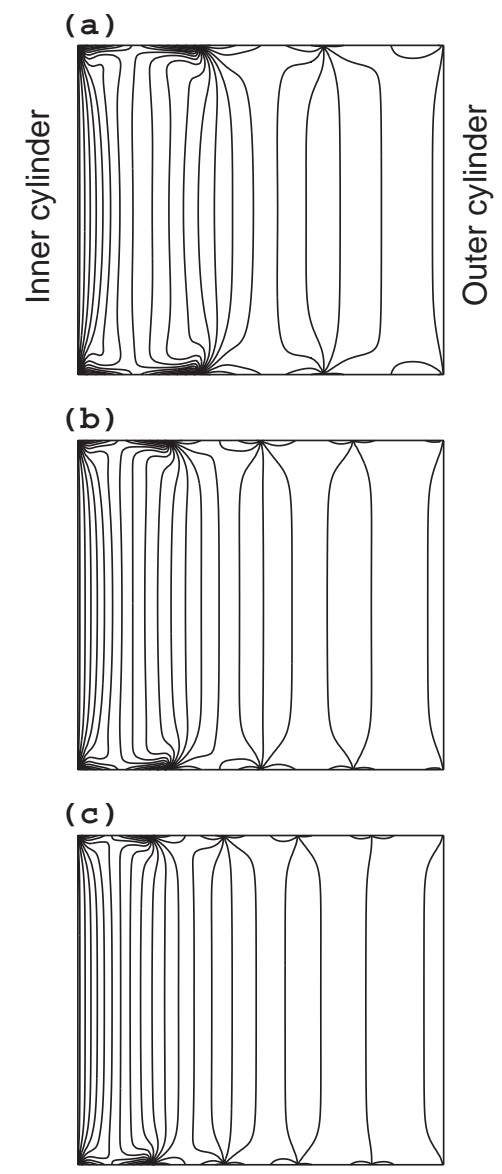

FIG. 19: Simulated toroidal velocity $v_{\varphi}$ for $R e=1600$ when the endcaps are divided into multiple rings; (a) 3 rings; (b) 4 rings; and (c) 5 rings.

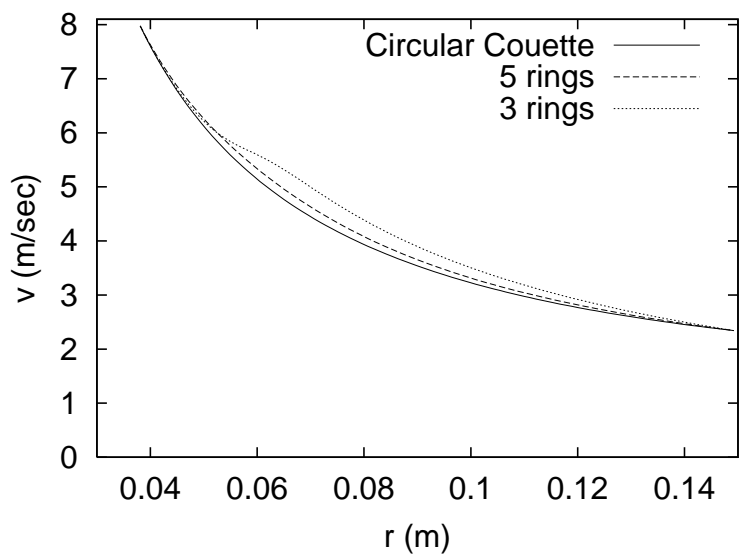

FIG. 20: Radial profile of $v_{\varphi}$ at the middle height when the dividing number of the cap is 3 and 5 . See also Fig. 19(b) and (c). 


\section{External Distribution}

Plasma Research Laboratory, Australian National University, Australia

Professor I.R. Jones, Flinders University, Australia

Professor João Canalle, Instituto de Fisica DEQ/IF - UERJ, Brazil

Mr. Gerson O. Ludwig, Instituto Nacional de Pesquisas, Brazil

Dr. P.H. Sakanaka, Instituto Fisica, Brazil

The Librarian, Culham Laboratory, England

Mrs. S.A. Hutchinson, JET Library, England

Professor M.N. Bussac, Ecole Polytechnique, France

Librarian, Max-Planck-Institut für Plasmaphysik, Germany

Jolan Moldvai, Reports Library, Hungarian Academy of Sciences, Central Research Institute for Physics, Hungary

Dr. P. Kaw, Institute for Plasma Research, India

Ms. P.J. Pathak, Librarian, Institute for Plasma Research, India

Ms. Clelia De Palo, Associazione EURATOM-ENEA, Italy

Dr. G. Grosso, Instituto di Fisica del Plasma, Italy

Librarian, Naka Fusion Research Establishment, JAERI, Japan

Library, Laboratory for Complex Energy Processes, Institute for Advanced Study, Kyoto University, Japan

Research Information Center, National Institute for Fusion Science, Japan

Dr. O. Mitarai, Kyushu Tokai University, Japan

Dr. Jiangang Li, Institute of Plasma Physics, Chinese Academy of Sciences, People's Republic of China

Professor Yuping Huo, School of Physical Science and Technology, People's Republic of China

Library, Academia Sinica, Institute of Plasma Physics, People's Republic of China

Librarian, Institute of Physics, Chinese Academy of Sciences, People's Republic of China

Dr. S. Mirnov, TRINITI, Troitsk, Russian Federation, Russia

Dr. V.S. Strelkov, Kurchatov Institute, Russian Federation, Russia

Professor Peter Lukac, Katedra Fyziky Plazmy MFF UK, Mlynska dolina F-2, Komenskeho Univerzita, SK-842 15 Bratislava, Slovakia

Dr. G.S. Lee, Korea Basic Science Institute, South Korea

Institute for Plasma Research, University of Maryland, USA

Librarian, Fusion Energy Division, Oak Ridge National Laboratory, USA

Librarian, Institute of Fusion Studies, University of Texas, USA

Librarian, Magnetic Fusion Program, Lawrence Livermore National Laboratory, USA

Library, General Atomics, USA

Plasma Physics Group, Fusion Energy Research Program, University of California at San Diego, USA

Plasma Physics Library, Columbia University, USA

Alkesh Punjabi, Center for Fusion Research and Training, Hampton University, USA

Dr. W.M. Stacey, Fusion Research Center, Georgia Institute of Technology, USA

Dr. John Willis, U.S. Department of Energy, Office of Fusion Energy Sciences, USA

Mr. Paul H. Wright, Indianapolis, Indiana, USA 
The Princeton Plasma Physics Laboratory is operated by Princeton University under contract with the U.S. Department of Energy.

\author{
Information Services \\ Princeton Plasma Physics Laboratory \\ P.O. Box 451 \\ Princeton, NJ 08543
}

Phone: 609-243-2750

Fax: 609-243-2751

e-mail: pppl_info@pppl.gov

Internet Address: http://www.pppl.gov 